\title{
On a new family of generalized Stirling and Bell numbers
}

\author{
Toufik Mansour \\ Department of Mathematics, University of Haifa, 31905 Haifa, Israel \\ toufik@math.haifa.ac.il \\ Matthias Schork \\ Camillo-Sitte-Weg 25, 60488 Frakfurt, Germany \\ mschork@member . ams .org \\ Mark Shattuck \\ Department of Mathematics, University of Haifa, 31905 Haifa, Israel \\ maarkons@excite.com \\ Submitted: Feb 11, 2011; Accepted: Mar 24, 2011; Published: Mar 31, 2011 \\ Mathematics Subject Classification: 05A15, 05A18, 05A19, 11B37, 11B73, 11B75
}

\begin{abstract}
A new family of generalized Stirling and Bell numbers is introduced by considering powers $(V U)^{n}$ of the noncommuting variables $U, V$ satisfying $U V=V U+h V^{s}$. The case $s=0$ (and $h=1$ ) corresponds to the conventional Stirling numbers of second kind and Bell numbers. For these generalized Stirling numbers, the recursion relation is given and explicit expressions are derived. Furthermore, they are shown to be connection coefficients and a combinatorial interpretation in terms of statistics is given. It is also shown that these Stirling numbers can be interpreted as $s$-rook numbers introduced by Goldman and Haglund. For the associated generalized Bell numbers, the recursion relation as well as a closed form for the exponential generating function is derived. Furthermore, an analogue of Dobinski's formula is given for these Bell numbers.
\end{abstract}

\section{Introduction}

The Stirling numbers (of first and second kind) are certainly among the most important combinatorial numbers as can be seen from their occurrence in many different contexts, see, e.g., $[6,14,35,38,42]$ and the references given therein. One of these interpretations is 
in terms of normal ordering special words in the Weyl algebra generated by the variables $U, V$ satisfying

$$
U V-V U=1
$$

where on the right-hand side the identity is denoted by 1. A concrete representation for (1) is given by the operators

$$
U \mapsto D \equiv \frac{d}{d x}, \quad V \mapsto X
$$

acting on a suitable space of functions (where $(X \cdot f)(x)=x f(x)$ ). In the mathematical literature, the simplification (i.e., normal ordering) of words in $D, X$ can be traced back to at least Scherk [31] (see [2] for a nice discussion of this and several other topics related to normal ordering words in $D, X)$ and many similar formulas have appeared in connection with operator calculus [6, 29, 30] and differential posets [37]. Already Scherk derived that the Stirling numbers of second kind $S(n, k)$ appear in the normal ordering of $(X D)^{n}$, or, in the variables used here,

$$
(V U)^{n}=\sum_{k=1}^{n} S(n, k) V^{k} U^{k} .
$$

This relation has been rediscovered countless times. In the physical literature, this connection was rediscovered by Katriel [17] in connection with normal ordering expressions in the boson annihilation $a$ and creation operator $a^{\dagger}$ satisfying the commutation relation $a a^{\dagger}-a^{\dagger} a=1$ of the Weyl algebra. Since the normal ordered form has many desirable properties simplifying many calculations, the normal ordering problem has been discussed in the physical literature extensively; see [2] for a thorough survey of the normal ordering for many functions of $X$ and $D$ with many references to the original literature. The relation (2) has been generalized by several authors to the form (here we assume $r \geq s$ )

$$
\left(V^{r} U^{s}\right)^{n}=V^{n(r-s)} \sum_{k=1}^{n} S_{r, s}(n, k) V^{k} U^{k},
$$

where the coefficients are, by definition, generalized Stirling numbers of second kind, see, e.g., $[3,5,9,19,20,22,23,25,32,40]$. Clearly, one has $S_{1,1}(n, k)=S(n, k)$. Let us briefly mention that also $q$-deformed versions of these Stirling numbers have been discussed $[22,23,32,40]$.

In another direction, Howard [16] unified many of the generalizations of the Stirling numbers by introducing degenerate weighted Stirling numbers $S(n, k, \lambda \mid \theta)$ which reduce for $\lambda=\theta=0$ to the conventional Stirling numbers of second kind, i.e., $S(n, k, 0 \mid 0)=S(n, k)$. He derived many properties of these numbers and also explicit expressions. The recursion relation for these numbers is given by [16, (4.11)]

$$
S(n+1, k, \lambda \mid \theta)=S(n, k-1, \lambda \mid \theta)+(k+\lambda-\theta n) S(n, k, \lambda \mid \theta) .
$$

As a last generalization of the Stirling and Bell numbers, we would like to mention [34] and the $r$-Stirling and $r$-Bell number (see [24] and the references therein). Neither of these two generalizations is directly related to the variant we discuss in the current paper. 
Two of the present authors considered in [21] the following generalization of the commutation relation (1), namely,

$$
U V-V U=h V^{s}
$$

where it was assumed that $h \in \mathbb{C} \backslash\{0\}$ and $s \in \mathbb{N}_{0}$. The parameter $h$ should be considered as a free "deformation parameter" (Planck's constant) and we will often consider the special case $h=1$. The dependance on the parameter $s$ will be central for the rest of the paper. Note that in the case $s=0$ (5) reduces to (1) (if $h=1$ ). Later on we will allow $s \in \mathbb{R}$, but first we restrict to $s \in \mathbb{N}_{0}$ to be able to use the above interpretation and the results of [21], where it was discussed that a concrete representation of (5) is given by the operators

$$
U \mapsto \mathcal{E}_{s} \equiv X^{s} D, \quad V \mapsto X .
$$

Now it is very natural to consider in the context of arbitrary $s \in \mathbb{N}_{0}$ the expression $(V U)^{n}$ for variables $U, V$ satisfying (5). In [21], the following result was derived:

Proposition 1.1. Let $V, U$ be variables satisfying (5) with $s \in \mathbb{N}_{0}$ and $h \in \mathbb{C} \backslash\{0\}$. Then one can define generalized Stirling numbers $\mathfrak{S}_{s ; h}(n, k)$ by

$$
(V U)^{n}=\sum_{k=1}^{n} \mathfrak{S}_{s ; h}(n, k) V^{s(n-k)+k} U^{k} .
$$

These generalized Stirling numbers can be expressed as

$$
\mathfrak{S}_{s ; h}(n, k)=h^{n-k} \sum_{l=k}^{n} S_{s+1,1}(n, l) s_{s, 1}(l, k),
$$

where $s_{s, 1}(l, k)$ are the generalized Stirling numbers of first kind introduced by Lang [19].

The coefficients $\mathfrak{S}_{s ; h}(n, k)$ can be interpreted as some kind of generalized Stirling numbers of second kind. As the explicit expression shows, they are very closely related to the generalized Stirling numbers $S_{r, 1}(n, k)$ considered by Lang [19, 20] - and already before him by Scherk [31], Carlitz [5] and Comtet [6, Page 220] - and more recently [2, 9, 26] (here one may also find a combinatorial interpretation of $S_{r, 1}(n, k)$ in terms of certain increasing trees). Burde considered in [4] matrices $X, A$ satisfying $X A-A X=X^{p}$ with $p \in \mathbb{N}$ and discussed the coefficients which appear upon normal ordering $(A X)^{n}$. He showed that they can be expressed for $p \geq 2$ through the degenerate weighted Stirling numbers $S(n, k, \lambda \mid \theta)$, where $\lambda=0$ and $\theta=\frac{p}{p-1}[4]$. Note that in terms of our variables $U, V$, Burde considered normal ordering $(U V)^{n}$, which is from our point of view less natural. However, since one can write $(V U)^{n}=V(U V)^{n-1} U$, these two problems are, of course, intimately related. Let us point out that Benaoum [1] considered the case $s=2$ of such variables in connection with a generalized binomial formula. This has been continued by Hagazi and Mansour [15], who considered special functions in such variables. More directly related to the present discussion, Diaz and Pariguan [8] described normal ordering in the meromorphic Weyl algebra. Recall that for $s=0$, one has the representation $D, X$ of the variables $U, V$ 
satisfying the relation $D X-X D=1$ of the Weyl algebra. Considering instead of $X$ the operator $X^{-1}$, one finds the relation $D\left(X^{-1}\right)-X^{-1} D=-X^{-2}$ and thus a representation of our variables $U, V$ for $s=2$ and $h=-1$. Considering $s=1$ (and $h=1$ ), one has a representation $V \mapsto X$ and $U \mapsto \mathcal{E}_{1}=X D$, the Euler operator, and the normal ordering is related to Touchard polynomials [7]. Varvak considered variables $U, V$ satisfying (5) for $s \in \mathbb{N}_{0}$ and their normal ordering and she pointed out the connection to $s$-rook numbers. As already mentioned above, Burde [4] considered combinatorial coefficients defined by a normal ordering of variables satisfying a very similar relation like (5).

As we will show in the present paper, the generalized Stirling numbers defined by (7) are very natural insofar as many properties of the conventional Stirling number of second kind find a simple analogue. For example, the interpretation of $S(n, k)$ as a rook number of a staircase Ferrers board generalizes in a beautiful fashion to the interpretation of $\mathfrak{S}_{s ; h}(n, k)$ as a $s$-rook number of the staircase board.

The corresponding generalized Bell numbers are introduced in analogy to the conventional case by

$$
\mathfrak{B}_{s ; h}(n):=\sum_{k=1}^{n} \mathfrak{S}_{s ; h}(n, k) .
$$

The structure of the paper is as follows. In Section 2, we consider the generalized Stirling and Bell numbers for $s=0$ and $s=1$ explicitly since many simplifications occur. For $s=0$, the generalized Stirling numbers are given by the conventional Stirling numbers of second kind, whereas in the case $s=1$, they are given by the unsigned Stirling numbers of first kind. In Section 3, the generalized Stirling numbers are considered for arbitrary $s \in \mathbb{R}$ and the recursion relation as well as an explicit formula is derived. The corresponding generalized Bell numbers are treated in Section 4, where the exponential generating function, the recursion relation and an analogue to Dobinski's formula are given. In Section 5, several combinatorial aspects of the generalized Stirling and Bell

numbers are treated. Furthermore, it is shown that the generalized Stirling numbers can be considered as connection coefficients and that they also have (for $s \in \mathbb{N}_{0}$ ) an interpretation in terms of $s$-rook numbers. Finally, in Section 6, some conclusions are presented.

\section{The generalized Stirling and Bell numbers for $s=$ 0,1}

In this section, we want to discuss the first two instances of the generalized Stirling and Bell numbers, namely, the cases $s=0$ and $s=1$.

\subsection{The case $s=0$}

Let $s=0$. Then the commutation relation (5) reduces nearly to (1) - only the factor $h$ remains. From this it is clear that the generalized Stirling numbers $\mathfrak{S}_{0 ; h}(n, k)$ are given 
by the conventional Stirling numbers of second kind,

$$
\mathfrak{S}_{0 ; h}(n, k)=h^{n-k} S(n, k),
$$

as was already discussed in [21] (and follows also immediately from Proposition 1.1). The generalized Bell numbers are, consequently, given by

$$
\mathfrak{B}_{0 ; h}(n)=\sum_{k=1}^{n} h^{n-k} S(n, k)
$$

and reduce, in the case $h=1$, to the usual Bell numbers, i.e., $\mathfrak{B}_{0 ; 1}(n)=\sum_{k=1}^{n} S(n, k)=$ $B(n)$.

\subsection{The case $s=1$}

Let $s=1$. The commutation relation (5) reduces in this case to $U V=V(U+h)$ and yields, after a small induction,

$$
U V^{k}=V^{k}(U+h k)
$$

This allows us to find the generalized Stirling numbers $\mathfrak{S}_{1 ; h}(n, k)$ in the following fashion. For $n=2$, we find $(V U)^{2}=V U V U=V^{2}(U+h) U$, where we have used (11) in the last step. Now it follows that

$$
(V U)^{3}=(V U)\left\{V^{2}(U+h) U\right\}=V\left(U V^{2}\right)(U+h) U=V^{3}(U+2 h)(U+h) U .
$$

An induction shows that, in general,

$$
(V U)^{n}=V^{n} \prod_{k=0}^{n-1}(U+k h)=V^{n} h^{n} \prod_{k=0}^{n-1}(\tilde{U}+k)
$$

where we have abbreviated $\tilde{U}=U / h$. Recalling the generating function of the signless Stirling numbers of first kind [38, Proposition 1.3.4]

$$
\sum_{k=0}^{n} c(n, k) y^{k}=y(y+1) \cdots(y+n-1)
$$

we can rewrite $(12)$ as

$$
(V U)^{n}=V^{n} h^{n} \sum_{k=0}^{n} c(n, k) \tilde{U}^{k}=\sum_{k=0}^{n} c(n, k) h^{n-k} V^{n} U^{k}
$$

A comparison with (7) shows that

$$
\mathfrak{S}_{1 ; h}(n, k)=h^{n-k} c(n, k)=(-h)^{n-k} s(n, k),
$$


where we have used the relation $s(n, k)=(-1)^{n-k} c(n, k)$ [38, Page 18]. The corresponding Bell numbers are, consequently, given by

$$
\mathfrak{B}_{1 ; h}(n)=\sum_{k=0}^{n} h^{n-k} c(n, k)=\sum_{k=0}^{n}(-h)^{n-k} s(n, k)
$$

and reduce, in the case $h=1$, to $\mathfrak{B}_{1 ; 1}(n)=\sum_{k=0}^{n} c(n, k)=n$ ! (which can be seen from (13) by considering $y=1$ ). Let us introduce the exponential generating function of the generalized Bell numbers by

$$
\mathfrak{B e}_{s ; h}(x):=\sum_{n \geq 0} \mathfrak{B}_{s ; h}(n) \frac{x^{n}}{n !} .
$$

Proposition 2.1. The exponential generating function of the generalized Bell numbers is given for $s=1$ and $h \in \mathbb{C} \backslash\{0\}$ by

$$
\mathfrak{B e}_{1 ; h}(x)=\frac{1}{(1-h x)^{1 / h}} .
$$

For $h=1$, it reduces to $\mathfrak{B e}_{1 ; 1}(x)=(1-x)^{-1}$.

Proof. Inserting the above expression $(15)$ for $\mathfrak{B}_{1 ; h}(n)$ into the definition of $\mathfrak{B e}_{1 ; h}(x)$ yields

$$
\mathfrak{B e}_{1 ; h}(x)=\sum_{n \geq 0} \sum_{k=0}^{n} h^{n-k} c(n, k) \frac{x^{n}}{n !}=\sum_{n, k \geq 0} c(n, k)\left(\frac{1}{h}\right)^{k} \frac{(h x)^{n}}{n !} .
$$

Recalling

$$
\sum_{n, k \geq 0} c(n, k) u^{k} \frac{z^{n}}{n !}=\frac{1}{(1-z)^{u}}
$$

the assertion follows.

\section{$3 \quad$ The generalized Stirling numbers for arbitrary $s$}

The following result (see [21]), which generalizes (11), will be useful in the subsequent computations.

Lemma 3.1. Let $U, V$ be variables satisfying (5) with $s \in \mathbb{N}_{0}$ and $h \in \mathbb{C} \backslash\{0\}$. Then one has for $k \in \mathbb{N}_{0}$ the relation

$$
U V^{k}=V^{k} U+h k V^{k-1+s} .
$$

Let us consider the first few generalized Stirling numbers explicitly. Clearly, $(V U)^{1}=$ $V U$, so $\mathfrak{S}_{s ; h}(1,1)=1$ (and, consequently, $\mathfrak{B}_{s ; h}(1)=1$ ). The first interesting case is $n=2$. Directly from the commutation relation and using (17), one finds

$$
(V U)^{2}=V U V U=V\left\{V U+h V^{s}\right\} U=V^{2} U^{2}+h V^{s+1} U,
$$


implying $\mathfrak{S}_{s ; h}(2,1)=h, \mathfrak{S}_{s ; h}(2,2)=1$ (and, consequently, $\mathfrak{B}_{s ; h}(2)=1+h$ ). The next case is slightly more tedious, but completely analogous,

$$
\begin{aligned}
(V U)^{3} & =V U\left\{V^{2} U^{2}+h V^{s+1} U\right\} \\
& =V\left\{U V^{2}\right\} U^{2}+h V\left\{U V^{s+1}\right\} U \\
& =V\left\{V^{2} U+h 2 V^{s+1}\right\} U^{2}+h V\left\{V^{s+1} U+h(s+1) V^{2 s}\right\} U \\
& =V^{3} U^{3}+3 h V^{s+2} U^{2}+h^{2}(s+1) V^{2 s+1} U,
\end{aligned}
$$

implying

$$
\mathfrak{S}_{s ; h}(3,1)=h^{2}(s+1), \quad \mathfrak{S}_{s ; h}(3,2)=3 h, \quad \mathfrak{S}_{s ; h}(3,3)=1
$$

and, consequently, $\mathfrak{B}_{s ; h}(3)=h^{2}(s+1)+3 h+1$.

As a first step, we now derive the recursion relation of the generalized Stirling numbers.

Proposition 3.2. The generalized Stirling numbers $\mathfrak{S}_{s ; h}(n, k)$ satisfy for $s \in \mathbb{N}_{0}$ and $h \in \mathbb{C} \backslash\{0\}$ the recursion relation

$$
\mathfrak{S}_{s ; h}(n+1, k)=\mathfrak{S}_{s ; h}(n, k-1)+h\{k+s(n-k)\} \mathfrak{S}_{s ; h}(n, k),
$$

with the initial value $\mathfrak{S}_{s ; h}(1,1)=1\left(\right.$ and $\mathfrak{S}_{s ; h}(n, 0)=\delta_{n, 0}$ for all $\left.n \in \mathbb{N}_{0}\right)$.

Proof. Instead of considering the explicit expression given in Proposition 1.1, we start from (7). On the one hand, we have $(V U)^{n+1}=\sum_{k=1}^{n+1} \mathfrak{S}_{s ; h}(n+1, k) V^{s(n+1-k)+k} U^{k}$. On the other hand, one has

$$
\begin{aligned}
(V U)^{n+1} & =\sum_{k=1}^{n} \mathfrak{S}_{s ; h}(n, k) V U V^{s(n-k)+k} U^{k} \\
& =\sum_{k=1}^{n} \mathfrak{S}_{s ; h}(n, k) V\left\{V^{s(n-k)+k} U+h(s(n-k)+k) V^{s(n-k)+k-1+s}\right\} U^{k} \\
& =\sum_{k=1}^{n} \mathfrak{S}_{s ; h}(n, k)\left\{V^{s(n-k)+k+1} U^{k+1}+h(s(n-k)+k) V^{s(n-k+1)+k} U^{k}\right\},
\end{aligned}
$$

where we have used (17) in the second line. Comparing the coefficients yields the asserted recursion relation.

Remark 3.3. As mentioned in Section 1, the generalized Stirling numbers $\mathfrak{S}_{s ; h}(n, k)$ are very closely related to the generalized Stirling numbers $S_{s, 1}(n, k)$. Lang [19, (13)] gives for them the following recursion relation (adapted to our notation)

$$
S_{s, 1}(n+1, k)=S_{s, 1}(n, k-1)+\{k+(s-1) n\} S_{s, 1}(n, k) .
$$

Comparing this to the recursion relation (4) of the degenerate weighted Stirling numbers $S(n, k, \lambda \mid \theta)$, one sees that choosing $\lambda=0$ and $\theta=-(s-1)=(1-s)$ reproduces the recursion relation of the $S_{s, 1}(n, k)$, i.e.,

$$
S_{s, 1}(n, k)=S(n, k, 0 \mid 1-s) .
$$

In contrast, the recursion relation (18) of the generalized Stirling numbers $\mathfrak{S}_{s ; h}(n, k)$ is not a special case of (4), although they look very similar. 
Example 3.1. Let $s=0$. The recursion relation (18) reduces to

$$
\mathfrak{S}_{0 ; h}(n+1, k)=\mathfrak{S}_{0 ; h}(n, k-1)+h k \mathfrak{S}_{0 ; h}(n, k),
$$

which is, in the case $h=1$, exactly the recursion relation of the Stirling numbers of second kind [38, Page 33]. In the case of arbitrary $h$, the generalized Stirling numbers are rescaled Stirling numbers of second kind, see (9).

Example 3.2. Let $s=1$. The recursion relation (18) reduces to

$$
\mathfrak{S}_{1 ; h}(n+1, k)=\mathfrak{S}_{1 ; h}(n, k-1)+h n \mathfrak{S}_{1 ; h}(n, k),
$$

which is, in the case $h=1$, exactly the recursion relation of the signless Stirling numbers of first kind [38, Lemma 1.3.3]. In the case of arbitrary $h$, the generalized Stirling numbers are rescaled signless Stirling numbers of first kind, see (14).

Now, although the recursion relation (18) was derived from the definition of the $\mathfrak{S}_{s, h}(n, k)$ in $(7)$ for $s \in \mathbb{N}_{0}$, we can now switch the point of view and define the generalized Stirling numbers for arbitrary $s \in \mathbb{R}$ by the recursion relation.

Definition 3.1. Let $s \in \mathbb{R}$ and $h \in \mathbb{C} \backslash\{0\}$. The generalized Stirling numbers $\mathfrak{S}_{s ; h}(n, k)$ are defined by the initial values and the recursion relation given in Proposition 3.2. The corresponding Bell numbers are then defined by (8).

It is interesting to note that, already in the case $s=2$, one obtains in the recursion relation $\mathfrak{S}_{2 ; h}(n+1, k)=\mathfrak{S}_{2 ; h}(n, k-1)+h(2 n-k) \mathfrak{S}_{2 ; h}(n, k)$ a nontrivial mix of $n$ and $k$ as factor in the second summand.

Example 3.3. Let $s=\frac{1}{2}$ and $h=2$. The corresponding generalized Stirling numbers satisfy the recursion relation

$$
\mathfrak{S}_{\frac{1}{2} ; 2}(n+1, k)=\mathfrak{S}_{\frac{1}{2} ; 2}(n, k-1)+\{n+k\} \mathfrak{S}_{\frac{1}{2} ; 2}(n, k),
$$

which is exactly the recursion relation of the (unsigned) Lah numbers $L(n, k)=\frac{n !}{k !}\left(\begin{array}{l}n-1 \\ k-1\end{array}\right)$ [6, Page 156], i.e.,

$$
\mathfrak{S}_{\frac{1}{2} ; 2}(n, k)=L(n, k) .
$$

Remark 3.4. Let us consider $h=1$. Then we can write (18) equivalently as

$$
\left.\mathfrak{S}_{s ; 1}(n+1, k)=\mathfrak{S}_{s ; 1}(n, k-1)+\{s n+(1-s) k)\right\} \mathfrak{S}_{s ; 1}(n, k) .
$$

Let us furthermore restrict to $s \in[0,1]$. Since $s=0$ corresponds to the conventional Stirling numbers of second kind $S(n, k)$ (see Example 3.1) and the case $s=1$ corresponds to the signless Stirling numbers of first kind $c(n, k)$ (see Example 3.2), one is tempted to view the generalized Stirling numbers $\mathfrak{S}_{s ; 1}(n, k)$ with $0<s<1$ due to the bracket in the second factor as some kind of "linear interpolation" (or "convex combination") between these two extremal points. 
Some special values of the generalized Stirling numbers can be obtained easily.

Proposition 3.5. The generalized Stirling numbers satisfy, for $n \geq 2$ and arbitrary $s \in \mathbb{R}$ and $h \in \mathbb{C} \backslash\{0\}$,

$$
\mathfrak{S}_{s ; h}(n, n)=1, \quad \mathfrak{S}_{s ; h}(n, n-1)=h\left(\begin{array}{l}
n \\
2
\end{array}\right), \quad \mathfrak{S}_{s ; h}(n, 1)=h^{n-1} \prod_{k=0}^{n-2}(1+k s) .
$$

In particular, one has for $s=2$ that $\mathfrak{S}_{2 ; h}(n, 1)=h^{n-1}(2 n-3) !$ !.

Proof. The recursion relation (18) shows that $\mathfrak{S}_{s ; h}(n, n)=\mathfrak{S}_{s ; h}(n-1, n-1)$ so that an induction together with $\mathfrak{S}_{s ; h}(1,1)=1$ yields the first assertion. The second follows also from the recursion relation by induction since

$\mathfrak{S}_{s ; h}(n, n-1)=\mathfrak{S}_{s ; h}(n-1, n-2)+h(n-1) \mathfrak{S}_{s ; h}(n-1, n-1)=\mathfrak{S}_{s ; h}(n-1, n-2)+h(n-1)$.

The last assertion follows from the recursion relation

$$
\mathfrak{S}_{s ; h}(n, 1)=h\{1+s(n-2)\} \mathfrak{S}_{s ; h}(n-1,1)
$$

and an induction.

In Table 1 the first few generalized Stirling numbers are given.

\begin{tabular}{r|r|r|r|r|r}
$n$ & $\mathfrak{S}_{s ; h}(n, 1)$ & $\mathfrak{S}_{s ; h}(n, 2)$ & $\mathfrak{S}_{s ; h}(n, 3)$ & $\mathfrak{S}_{s ; h}(n, 4)$ & $\mathfrak{S}_{s ; h}(n, 5)$ \\
\hline \hline 1 & 1 & & & & \\
2 & $h$ & 1 & & & \\
3 & $h^{2}(s+1)$ & $3 h$ & 1 & & \\
4 & $h^{3}(s+1)(2 s+1)$ & $h^{2}(4 s+7)$ & $6 h$ & 1 & \\
5 & $h^{4}(s+1)(2 s+1)(3 s+1)$ & $h^{3}\left(10 s^{2}+25 s+15\right)$ & $h^{2}(10 s+25)$ & $10 h$ & 1
\end{tabular}

Table 1: The first few generalized Stirling numbers $\mathfrak{S}_{s ; h}(n, k)$.

For later use, we introduce the exponential generating function of the generalized Stirling numbers with $k=1$, i.e., of $\mathfrak{S}_{s ; h}(n, 1)$ by

$$
\mathfrak{S e}_{s ; h}(x):=\sum_{n \geq 1} \mathfrak{S}_{s ; h}(n, 1) \frac{x^{n}}{n !} .
$$

Proposition 3.6. Let $s \in \mathbb{R} \backslash\{0,1\}$ and $h \in \mathbb{C} \backslash\{0\}$. The function $\mathfrak{S e}_{s ; h}$ satisfies the differential equation

$$
\mathfrak{S e}_{s ; h}^{\prime}(x)=\frac{1}{(1-h s x)^{\frac{1}{s}}} .
$$

Consequently, it is given explicitly by

$$
\mathfrak{S}_{s ; h}(x)=\frac{1}{h(s-1)}\left\{1-(1-h s x)^{\frac{s-1}{s}}\right\} .
$$


In the case $s=0$, it is given by

$$
\mathfrak{S}_{0 ; h}(x)=\frac{1}{h}\left(e^{h x}-1\right) .
$$

In the case $s=1$, it is given by

$$
\mathfrak{S}_{1 ; h}(x)=\log \left(\frac{1}{(1-h x)^{1 / h}}\right) .
$$

Proof. Let us consider first the case $s \neq 0,1$. Using the binomial series, we obtain

$$
\frac{1}{(1-h s x)^{\frac{1}{s}}}=\sum_{m \geq 0}\left(\begin{array}{c}
m+\frac{1}{s}-1 \\
m
\end{array}\right) m !(h s)^{m} \frac{x^{m}}{m !} .
$$

The asserted differential equation follows due to

$$
\left(\begin{array}{c}
m+\frac{1}{s}-1 \\
m
\end{array}\right) m !(h s)^{m}=h^{m} \prod_{j=0}^{m-1}(1+j s)=\mathfrak{S}_{s ; h}(m+1,1)
$$

where we have used in the second equation, Proposition 3.5. The explicit form of the exponential generating function follows from

$$
\mathfrak{S} \mathfrak{e}_{s ; h}(x)=\int_{0}^{x} \frac{d t}{(1-h s t)^{\frac{1}{s}}}
$$

by a standard integration. Let us turn to the case $s=0$. Using $(9)$, one finds $\mathfrak{S}_{0 ; h}(n, 1)=$ $h^{n-1} S(n, 1)=h^{n-1}$ and, consequently, $\mathfrak{S e}_{0 ; h}(x)=\sum_{n>1} h^{n-1} \frac{x^{n}}{n !}=\frac{1}{h}\left(e^{h x}-1\right)$. In the case $s=1$, we use in a similar fashion (14) and find $\mathfrak{S}_{1 ; h}(n, \overline{1})=(-h)^{n-1} s(n, 1)=h^{n-1}(n-1)$ !, implying

$$
\mathfrak{S}_{1 ; h}(x)=\sum_{n \geq 1} h^{n-1}(n-1) ! \frac{x^{n}}{n !}=\frac{1}{h} \sum_{n \geq 1} \frac{(h x)^{n}}{n}=\frac{1}{h} \log \left(\frac{1}{1-h x}\right)=\log \left(\frac{1}{(1-h x)^{1 / h}}\right)
$$

as asserted.

Example 3.4. Let $h=1$ and $s=2$. It follows from Proposition 3.6 that

$$
\mathfrak{S}_{2 ; 1}(x)=1-\sqrt{1-2 x} .
$$

According to Example 5.2.6 on page 15 of [39], this is the exponential generating function of binary set bracketings such that if $b(n)$ is the number of (unordered) complete binary trees with $n$ labeled endpoints, one has $\sum_{n \geq 0} b(n) \frac{x^{n}}{n !}=1-\sqrt{1-2 x}$. Thus, $\mathfrak{S}_{2 ; 1}(n, 1)=$ $b(n)$. Since $b(n)=1 \cdot 3 \cdot 5 \cdots(2 n-3)=(2 n-\overline{3}) !$ !, this is in accordance with Proposition 3.5. 
Remark 3.7. Recall that for the conventional Stirling numbers of second kind - i.e., the case $s=0$ and $h=1$ - if one considers the ordinary generating function $B_{k}(x):=$ $\sum_{n \geq k} S(n, k) x^{n}$, and applies the two-term recurrence for $S(n, k)$, then one obtains the relation $B_{k}(x)=x B_{k-1}(x)+k x B_{k}(x)$, or,

$$
(1-k x) B_{k}(x)=x B_{k-1}(x) .
$$

This can be solved easily for $B_{k}(x)$, allowing for a determination of the parity (i.e., congruence modulo 2) of $S(n, k)$ [42, Page 149]. For the case of arbitrary s, the same procedure is not successful due to the mixing of $n$ and $k$ in the second factor of (18). Introducing $B_{k \mid s ; h}:=\sum_{n \geq k} \mathfrak{S}_{s ; h}(n, k) x^{n}$, the recursion (18) yields

$$
(1-h(1-s) k x) B_{k \mid s ; h}(x)=x B_{k-1 \mid s ; h}(x)+h s x^{2} B_{k \mid s ; h}^{\prime}(x) .
$$

Clearly, for $s=0$ and $h=1$, one has $B_{k \mid 0 ; 1}(x)=B_{k}(x)$ and this equation reduces to the one for the conventional Stirling numbers given above, but in general it seems much harder to solve.

Let us introduce the bivariate ordinary generating function of the generalized Stirling numbers by

$$
B_{s ; h}(x, y):=\sum_{k \geq 0} B_{k \mid s ; h}(x) y^{k}=\sum_{k \geq 0} \sum_{n \geq k} \mathfrak{S}_{s ; h}(n, k) x^{n} y^{k}
$$

Proposition 3.8. Fix $h \neq 0$. For $s \in \mathbb{R}$ the bivariate ordinary generating function $B_{s ; h}(x, y)$ satisfies the partial differential equation

$$
\left\{s x \frac{\partial}{\partial x}+(1-s) y \frac{\partial}{\partial y}\right\} B_{s ; h}(x, y)=\left(\frac{1-x y}{h x}\right) B_{s ; h}(x, y) .
$$

Proof. From (19), one obtains, upon multiplying by $y^{k}$ and summing over $k$, the partial differential equation

$$
\left\{1-h(1-s) x y \frac{\partial}{\partial y}\right\} B_{s ; h}(x, y)=x y B_{s ; h}(x, y)+h s x^{2} \frac{\partial}{\partial x} B_{s ; h}(x, y),
$$

which is equivalent to the asserted equation.

Example 3.5. Note that (20) reduces in the case $s=0$ and $h=1$ - corresponding to the conventional Stirling numbers of second kind (see Example 3.1) - to

$$
\frac{\partial B_{0 ; 1}(x, y)}{\partial y}=\left(\frac{1-x y}{x y}\right) B_{0 ; 1}(x, y)
$$

Considering instead $s=1$ and $h=1$ - corresponding to the unsigned Stirling numbers of first kind (see Example 3.2) - yields

$$
\frac{\partial B_{1 ; 1}(x, y)}{\partial x}=\left(\frac{1-x y}{x^{2}}\right) B_{1 ; 1}(x, y) .
$$

The ELECTROnic Journal of COMBinatorics 18 (2011), \#P77 
Finally, letting $s=\frac{1}{2}$ and $h=2$ - corresponding to the unsigned Lah numbers (see Example 3.3) - we obtain from (20) for the bivariate ordinary generating function of the unsigned Lah numbers

$$
\left\{x \frac{\partial}{\partial x}+y \frac{\partial}{\partial y}\right\} B_{\frac{1}{2} ; 2}(x, y)=\left(\frac{1-x y}{x}\right) B_{\frac{1}{2} ; 2}(x, y) .
$$

Now we give the explicit form of the generalized Stirling numbers in the following theorem.

Theorem 3.9. Fix $h \neq 0$. For $s \in \mathbb{R} \backslash\{0,1\}$, the generalized Stirling numbers are given explicitly by

$$
\mathfrak{S}_{s ; h}(n, k)=\frac{h^{n-k} s^{n} n !}{(1-s)^{k} k !} \sum_{j=0}^{k}(-1)^{k-j}\left(\begin{array}{c}
k \\
j
\end{array}\right)\left(\begin{array}{c}
n+\frac{j}{s}-j-1 \\
n
\end{array}\right),
$$

for all $n \geq k \geq 0$. If $s=0$, then $\mathfrak{S}_{0 ; h}(n, k)=h^{n-k} S(n, k)$, and if $s=1$, then $\mathfrak{S}_{1 ; h}(n, k)=$ $(-h)^{n-k} s(n, k)$.

Proof. Let $a=h s$ and $b=h-h s$. For convenience, rename $\mathfrak{S}_{s ; h}(n, k)$ as $\mathfrak{S}_{a ; b}(n, k)$. Then (18) may be rewritten as

$$
\mathfrak{S}_{a ; b}(n, k)=\mathfrak{S}_{a ; b}(n-1, k-1)+[a(n-1)+b k] \mathfrak{S}_{a ; b}(n-1, k), \quad n \geq k \geq 1,
$$

with $\mathfrak{S}_{a ; b}(0,0)=1$ and $\mathfrak{S}_{a ; b}(n, k)=0$ if $0 \leq n<k$. Define the exponential generating function $L_{k}(x)$ for $k \geq 0$ by

$$
L_{k}(x):=\sum_{n \geq k} \mathfrak{S}_{a ; b}(n, k) \frac{x^{n}}{n !}
$$

Multiplying both sides of (21) by $\frac{x^{n}}{n !}$, summing over $n$ and then differentiating with respect to $x$, we obtain

$$
L_{k}^{\prime}(x)-\frac{b k}{1-a x} L_{k}(x)=\frac{L_{k-1}(x)}{1-a x}, \quad k \geq 1,
$$

with $L_{0}(x)=1$.

The case $s \neq 0,1$ : Now assume that $a, b \neq 0$ (we treat the cases $a=0$ or $b=0$ below). Multiplying both sides of $(22)$ by $(1-a x)^{\frac{b k}{a}}$, we see that $(22)$ may be expressed as

$$
\left[(1-a x)^{\frac{b k}{a}} L_{k}(x)\right]^{\prime}=(1-a x)^{\frac{b}{a}-1} \times(1-a x)^{\frac{b(k-1)}{a}} L_{k-1}(x) .
$$

Letting $r:=\frac{b}{a}-1$ and $h_{k}(x):=(1-a x)^{\frac{b k}{a}} L_{k}(x), k \geq 0$, this equation may be rewritten as

$$
h_{k}^{\prime}(x)=(1-a x)^{r} h_{k-1}(x)
$$


with $h_{0}(x)=1$. To find $h_{k}(x)$, and thus $\left.L_{k}(x)=(1-a x)^{-\frac{b k}{a}} h_{k}(x)\right)$, we consider the further generating function

$$
h(x, y):=\sum_{k \geq 0} h_{k}(x) y^{k}
$$

From equation (23), we obtain

$$
\frac{\partial}{\partial x} h(x, y)=(1-a x)^{r} y h(x, y)
$$

with $h(x, 0)=h(0, y)=1$, which leads to

$$
h(x, y)=e^{\frac{1-(1-a x)^{r+1}}{a(r+1)} y}=\sum_{k \geq 0} \frac{\left(1-(1-a x)^{r+1}\right)^{k}}{a^{k}(r+1)^{k}} \frac{y^{k}}{k !} .
$$

Thus, by $r=\frac{b}{a}-1$,

$$
L_{k}(x)=\frac{h_{k}(x)}{(1-a x)^{\frac{b k}{a}}}=\frac{\left(1-(1-a x)^{r+1}\right)^{k}}{a^{k}(r+1)^{k} k !(1-a x)^{\frac{b k}{a}}}=\sum_{j=0}^{k}(-1)^{j}\left(\begin{array}{c}
k \\
j
\end{array}\right) \frac{1}{b^{k} k !(1-a x)^{\frac{b(k-j)}{a}}} .
$$

Hence, by comparing the $x^{n}$ coefficient on both sides of the above equation, we obtain

$$
\mathfrak{S}_{a ; b}(n, k)=\frac{n !}{b^{k} k !} \sum_{j=0}^{k}(-1)^{j}\left(\begin{array}{l}
k \\
j
\end{array}\right)\left(\begin{array}{c}
n+\frac{b(k-j)}{a}-1 \\
n
\end{array}\right) a^{n} .
$$

Substituting $a=h s$ and $b=h-h s$ yields the desired result.

The case $s=0$ : We now treat the case $s=0$, i.e., $a=0$ and $b \neq 0$. Taking $a=0$ in (22), we get

$$
L_{k}^{\prime}(x)-b k L_{k}(x)=L_{k-1}(x)
$$

which is equivalent to

$$
\left(e^{-b k x} L_{k}(x)\right)^{\prime}=e^{-b k x} L_{k-1}(x)=e^{-b x} \cdot e^{-b(k-1) x} L_{k-1}(x),
$$

with $L_{0}(x)=1$. Define

$$
d_{k}(x):=e^{-b k x} L_{k}(x)
$$

So

$$
d_{k}^{\prime}(x)=e^{-b x} d_{k-1}(x), \quad k \geq 1,
$$

with $d_{0}(x)=1$. Multiplying this recurrence by $y^{k}$ and summing over $k \geq 1$, we obtain

$$
\frac{\partial}{\partial x} d(x, y)=e^{-b x} y d(x, y)
$$

where we have defined

$$
d(x, y):=\sum_{k \geq 0} d_{k}(x) y^{k}
$$


Solving this equation, noting the boundary conditions $d(0, y)=d(x, 0)=1$, we obtain

$$
d(x, y)=e^{\frac{y}{b}\left(1-e^{-b x}\right)} .
$$

Hence, $d_{k}(x)=\left[y^{k}\right] d(x, y)=\frac{\left(1-e^{-b x}\right)^{k}}{b^{k} k !}$, which implies

$$
L_{k}(x)=\frac{\left(1-e^{-b x}\right)^{k}}{e^{-b k x} b^{k} k !}=\frac{\left(e^{b x}-1\right)^{k}}{b^{k} k !} .
$$

Substituting $a=0$ and $b=h$, this shows that

$$
\mathfrak{S}_{0 ; h}(n, k)=n !\left[x^{n}\right] L_{k}(x)=n !\left[x^{n}\right] \frac{\left(e^{h x}-1\right)^{k}}{h^{k} k !}=\frac{n !}{h^{k}}\left[x^{n}\right] \sum_{m \geq 0} S(m, k) \frac{(h x)^{m}}{m !}=h^{n-k} S(n, k),
$$

as requested.

The case $s=1$ : We now treat the case $s=1$, i.e., $a \neq 0, b=0$, and $r=-1$. Taking $r=-1$ in (24), we obtain

$$
\frac{\partial}{\partial x} h(x, y)=\frac{y}{1-a x} h(x, y)
$$

with $h(0, y)=h(x, 0)=1$. Solving this equation yields

$$
h(x, y)=(1-a x)^{-\frac{y}{a}}=\sum_{n \geq 0}\left(\begin{array}{c}
n+\frac{y}{a}-1 \\
n
\end{array}\right)(a x)^{n} .
$$

Thus,

$$
\begin{aligned}
{\left[x^{n}\right] h(x, y) } & =a^{n}\left(\begin{array}{c}
n+\frac{y}{a}-1 \\
n
\end{array}\right)=(-a)^{n}\left(\begin{array}{c}
-\frac{y}{a} \\
n
\end{array}\right) \\
& =(-a)^{n} \frac{\left(-\frac{y}{a}\right)\left(-\frac{y}{a}-1\right) \cdots\left(-\frac{y}{a}-n+1\right)}{n !} \\
& =\frac{a^{n}}{n !} \prod_{j=0}^{n-1}(\tilde{y}+j),
\end{aligned}
$$

where we have abbreviated $\tilde{y}=y / a$. Recalling (13), it follows that

$$
n !\left[x^{n}\right] h(x, y)=a^{n} \sum_{k \geq 0} c(n, k) \tilde{y}^{k}=\sum_{k \geq 0} c(n, k) a^{n-k} y^{k}
$$

Substituting $a=h$ gives

$$
\mathfrak{S}_{1 ; h}(n, k)=h^{n-k} c(n, k)=(-h)^{n-k} s(n, k),
$$

which completes the proof. 
Remark 3.10. During the proof of the theorem, we considered the cases $s=0$ and $s=1$ explicitly using generating function techniques. However, we already showed that the generalized Stirling numbers are given for $s=0$ by $\mathfrak{S}_{0 ; h}(n, k)=h^{n-k} S(n, k)$ in (9) and for $s=1$ by $\mathfrak{S}_{1 ; h}(n, k)=(-h)^{n-k} s(n, k)$ in $(14)$.

We now want to give an equivalent expression for the generalized Stirling numbers making the analogy to the conventional Stirling numbers of second kind $S(n, k)$ closer. Recall that

$$
S(n, k)=\frac{1}{k !} \sum_{r=0}^{k}(-1)^{k-r}\left(\begin{array}{l}
k \\
r
\end{array}\right) r^{n} .
$$

Corollary 3.11. Let $s \in \mathbb{R} \backslash\{0,1\}$ and $h \in \mathbb{C} \backslash\{0\}$. The generalized Stirling numbers can be written as

$$
\mathfrak{S}_{s ; h}(n, k)=\frac{h^{n-k}}{k !} \sum_{r=0}^{k}(-1)^{k-r}\left(\begin{array}{l}
k \\
r
\end{array}\right) \psi_{s}(n, k ; r),
$$

where the function $\psi_{s}(n, k ; r)$ is defined by

$$
\psi_{s}(n, k ; r):=\sum_{l=0}^{n} c(n, l) \frac{s^{n-l}}{(1-s)^{k-l}} r^{l} .
$$

Proof. Starting from the explicit expression derived in Theorem 3.9, we can write

$$
\mathfrak{S}_{s ; h}(n, k)=\frac{h^{n-k}}{k !} \sum_{r=0}^{k}(-1)^{k-r}\left(\begin{array}{l}
k \\
r
\end{array}\right)\left(\begin{array}{c}
n+\frac{r}{s}-r-1 \\
n
\end{array}\right) \frac{n ! s^{n}}{(1-s)^{k}} .
$$

Using (13), we obtain

$$
\left(\begin{array}{c}
n+\frac{r}{s}-r-1 \\
n
\end{array}\right)=\frac{1}{n !} \prod_{l=0}^{n-1}\left(r\left(\frac{1-s}{s}\right)+l\right)=\frac{1}{n !} \sum_{l=0}^{n} c(n, l) \frac{(1-s)^{l}}{s^{l}} r^{l} .
$$

Inserting this and using the definition of $\psi_{s}(n, k ; r)$, the assertion follows.

It is interesting to consider formally $s \rightarrow 0$. Since $\frac{s^{n-l}}{(1-s)^{k-l}} \rightarrow \delta_{n-l, 0}$ and $c(n, n)=1$, one obtains $\psi_{s}(n, k ; r) \rightarrow r^{n}$, showing

$$
\mathfrak{S}_{s ; h}(n, k) \stackrel{s \rightarrow 0}{\longrightarrow} h^{n-k} S(n, k) .
$$

Note that the consideration $s \rightarrow 1$ is more difficult since $\psi_{s}(n, k ; r)$ has singularities for $s \rightarrow 1$.

Example 3.6. Let us consider the generalized Stirling numbers for $h=1$ and $s=-\frac{1}{r}$ with $r \in \mathbb{N}$. It follows that

$$
\begin{aligned}
\mathfrak{S}_{-\frac{1}{r} ; 1}(n, k) & =\frac{(-1)^{n} n !}{r^{n-k}(r+1)^{k} k !} \sum_{j=0}^{k}(-1)^{k-j}\left(\begin{array}{c}
k \\
j
\end{array}\right)\left(\begin{array}{c}
n-(r+1) j-1 \\
n
\end{array}\right) \\
& =\frac{1}{r^{n-k}(r+1)^{k}} \sum_{j=0}^{k} \frac{(-1)^{k-j}\{(r+1) j\}^{\underline{n}}}{j !(k-j) !},
\end{aligned}
$$


where we have used the lower factorial $m^{\underline{k}}=m(m-1)(m-2) \cdots(m-k+1)$. For $r=1$, this reduces to

$$
\mathfrak{S}_{-1 ; 1}(n, k)=\frac{1}{2^{k}} \sum_{j=0}^{k} \frac{(-1)^{k-j}(2 j)^{\underline{n}}}{j !(k-j) !} .
$$

From (18), one has

$$
\mathfrak{S}_{-1 ; 1}(n+1, k)=\mathfrak{S}_{-1 ; 1}(n, k-1)+(2 k-n) \mathfrak{S}_{-1 ; 1}(n, k) .
$$

Remark 3.12. It would be interesting to consider the asymptotic behavior of the generalized Stirling numbers. However, for general $h$ and $s$, this seems to be difficult, so we restrict to the case $h=1$ and $s>1$. Considering the recursion relation for large $n$, one sees that for fixed $k$ the largest quotient $\frac{\mathfrak{S}_{s ; 1}(n+1, k)}{\mathfrak{S}_{s ; 1}(n, k)}$ results by choosing $k=1$. Furthermore, considering explicit values for the generalized Stirling numbers shows that, already for relatively small $n$, the largest value of the $\mathfrak{S}_{s ; 1}(n, k)$ is attained for $k=1$ and yields the greatest contribution to the Bell number $\mathfrak{B}_{s ; 1}(n)$. From the explicit expression given in Proposition 3.5, one has for large $n$ that $\mathfrak{S}_{s ; 1}(n, 1) \sim s(n-2) \mathfrak{S}_{s ; 1}(n-1,1)$. Clearly, this can be iterated, showing that one has the rough estimate $\mathfrak{S}_{s ; 1}(n, 1) \geq s^{l} \frac{(n-2) !}{(n-2-l) !} \mathfrak{S}_{s ; 1}(n-l, 1)$. Choosing $l=n-3$ yields $\mathfrak{S}_{s ; 1}(n, 1) \geq(1+s) s^{n-3}(n-2)$ !. However, for small $n$, the assumption made becomes worse, so one should instead use a smaller l, e.g., $l=n-6$. Using this, one obtains for $n>6$ a very rough estimate

$$
\mathfrak{S}_{s ; 1}(n, 1) \geq \frac{(1+s)(1+2 s)(1+3 s)(1+4 s)}{24} s^{n-6}(n-2) ! .
$$

The heuristics mentioned above are made explicit in the following conjecture.

Conjecture 3.13. Let $h=1$ and $s>1$. The sequence of generalized Stirling numbers $\left\{\mathfrak{S}_{s ; 1}(n, k)\right\}_{k=1}^{n}$ is unimodal for every $n \geq 1$. Furthermore, for $s \geq 2$ the sequence is monotone decreasing for every $n \geq 1$ and for $s>3$ the sequence is strictly monotone decreasing for $n \geq 3$.

\section{The generalized Bell numbers for arbitrary $s$}

In this section, we discuss the generalized Bell numbers. Define

$$
L_{s ; h}(x, y):=\sum_{k \geq 0} \sum_{n \geq k} \mathfrak{S}_{s ; h}(n, k) \frac{x^{n} y^{k}}{n !} .
$$

Note that one obtains for $y=1$, by the definition of the generalized Bell numbers, that

$$
L_{s ; h}(x, 1)=\sum_{k \geq 0} \sum_{n \geq k} \mathfrak{S}_{s ; h}(n, k) \frac{x^{n}}{n !}=\sum_{n \geq 0} \mathfrak{B}_{s ; h}(n) \frac{x^{n}}{n !}=\mathfrak{B e}_{s ; h}(x),
$$

i.e., the exponential generating function of the generalized Bell numbers. From the proof of Theorem 3.9, we obtain the following explicit formulas for the generating functions $L_{s ; h}(x, y)$. 
Corollary 4.1. Fix $h \neq 0$. If $s \in \mathbb{R} \backslash\{0,1\}$, then

$$
L_{s ; h}(x, y)=e^{\left\{1-(1-h s x)^{\frac{s-1}{s}}\right\} \frac{y}{h(s-1)}} .
$$

If $s=0$, then

$$
L_{0 ; h}(x, y)=e^{\frac{y}{h}\left(e^{h x}-1\right)} .
$$

If $s=1$, then

$$
L_{1 ; h}(x, y)=(1-h x)^{-\frac{y}{h}} .
$$

Proof. If $s \neq 0,1$, then $a, b \neq 0$, where $a=h s$ and $b=h-h s$, as defined above. By the proof of Theorem 3.9, we have

$$
L_{k}(x)=\frac{\left(\frac{1}{(1-a x)^{b / a}}-1\right)^{k}}{k ! b^{k}} .
$$

Thus,

$$
L_{s ; h}(x, y)=\sum_{k \geq 0} L_{k}(x) y^{k}=e^{\left(\frac{1}{\left.(1-a x)^{\frac{b}{a}}-1\right)}\right)^{\frac{y}{b}}},
$$

which gives the first formula (after substituting $a=h s, b=h-h s$ and several simplifications). If $s=0$, then $L_{k}(x)$ was determined to be

$$
L_{k}(x)=\frac{\left(e^{h x}-1\right)^{k}}{h^{k} k !},
$$

implying the desired formula. Similarly, if $s=1$, then $L_{1 ; h}(x, y)$ was denoted in the proof by $h(x, y)$ and already given in $(27)$.

Taking $y=1$ in the prior corollary yields the exponential generating function for the generalized Bell numbers and, therefore, leads to explicit Dobinski-type formulas for the $n$-th generalized Bell numbers $\mathfrak{B}_{s ; h}(n)$.

Corollary 4.2. Fix $h \neq 0$. If $s \in \mathbb{R} \backslash\{0,1\}$, then

$$
\mathfrak{B}_{s ; h}(n)=n !(-h s)^{n} e^{\frac{1}{h(s-1)}} \sum_{j \geq 0}\left(\begin{array}{c}
\frac{(s-1) j}{s} \\
n
\end{array}\right) \frac{1}{j ! h^{j}(1-s)^{j}} .
$$

If $s=0$, then

$$
\mathfrak{B}_{0 ; h}(n)=\frac{1}{e^{\frac{1}{h}}} \sum_{j \geq 0} \frac{h^{n-j} j^{n}}{j !}
$$

If $s=1$, then

$$
\mathfrak{B}_{1 ; h}(n)=\prod_{j=0}^{n-1}(1+j h)=\sum_{j=0}^{n} h^{n-j} c(n, j) .
$$


Proof. First suppose $s \neq 0,1$. Taking $y=1$ in $L_{s ; h}(x, y)$ gives

$$
\begin{aligned}
L_{s ; h}(x, 1) & =e^{\frac{1}{h(s-1)}} e^{\frac{(1-h s x) \frac{s-1}{s}}{h(1-s)}}=e^{\frac{1}{h(s-1)}} \sum_{j \geq 0} \frac{1}{j ! h^{j}(1-s)^{j}}(1-h s x)^{\frac{(s-1) j}{s}} \\
& =e^{\frac{1}{h(s-1)}} \sum_{j \geq 0} \sum_{k \geq 0} \frac{1}{j ! h^{j}(1-s)^{j}}\left(\begin{array}{c}
\frac{(s-1) j}{s} \\
k
\end{array}\right)(-h s x)^{k},
\end{aligned}
$$

which implies, due to $L_{s ; h}(x, 1)=\mathfrak{B e}_{s ; h}(x)$, that

$$
\mathfrak{B}_{s ; h}(n)=n !\left[x^{n}\right] L_{s ; h}(x, 1)=n ! e^{\frac{1}{h(s-1)}} \sum_{j \geq 0} \frac{1}{j ! h^{j}(1-s)^{j}}\left(\begin{array}{c}
\frac{(s-1) j}{s} \\
n
\end{array}\right)(-h s)^{n},
$$

completing the first case. If $s=0$, we have

$$
\mathfrak{B}_{0 ; h}(n)=n !\left[x^{n}\right] L_{0 ; h}(x, 1)=n ! e^{-\frac{1}{h}}\left[x^{n}\right] \sum_{j \geq 0} \frac{1}{j !}\left(\frac{e^{h x}}{h}\right)^{j}=n ! e^{-\frac{1}{h}} \sum_{j \geq 0} \frac{1}{h^{j} j !} \frac{(h j)^{n}}{n !},
$$

showing the assertion. The third case follows similarly by noting

$$
\mathfrak{B}_{1 ; h}(n)=n !\left[x^{n}\right] L_{1 ; h}(x, 1)=n !\left[x^{n}\right](1-h x)^{-\frac{1}{h}}=n !\left[x^{n}\right] \sum_{n \geq 0}\left(\begin{array}{c}
n+\frac{1}{h}-1 \\
n
\end{array}\right)(h x)^{n} .
$$

Thus,

$$
\mathfrak{B}_{1 ; h}(n)=n ! h^{n}\left(\begin{array}{c}
n+\frac{1}{h}-1 \\
n
\end{array}\right)=n ! h^{n} \frac{1(1+h)(1+2 h) \cdots(1+(n-1) h)}{n ! h^{n}}=\prod_{j=0}^{n-1}(1+j h) .
$$

Using (13) yields the second asserted form of the generalized Bell numbers $\mathfrak{B}_{1 ; h}(n)$, which was already given in (15) and which is equivalent to the definition.

Corollary 4.3. Fix $h \neq 0$ and let $s \in \mathbb{R} \backslash\{0,1\}$. The generalized Bell numbers can also be written as

$$
\mathfrak{B}_{s ; h}(n)=e^{\frac{1}{h(s-1)}} \sum_{j \geq 0} \frac{(h(1-s))^{n-j} j^{n}}{j !} \prod_{k=0}^{n-1}\left\{1-\frac{k s}{j(s-1)}\right\} .
$$

Proof. Use the expression for the generalized Bell numbers given in Corollary 4.2 and expand the binomial coefficient.

Note that considering $s=0$ and $h=1$ yields the well-known classical Dobinski relation,

$$
\mathfrak{B}_{0 ; 1}(n)=\frac{1}{e} \sum_{j \geq 0} \frac{j^{n}}{j !} .
$$

In Table 2 the first few generalized Bell numbers are given. 


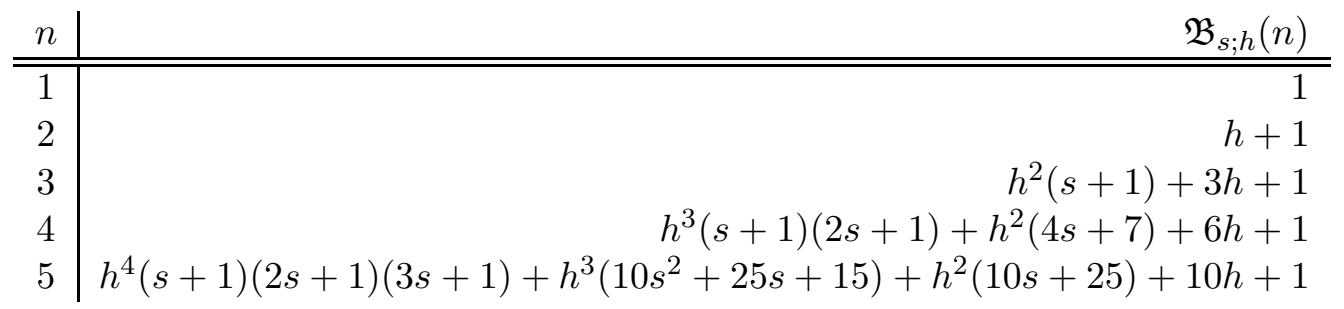

Table 2: The first few generalized Bell numbers $\mathfrak{B}_{s ; h}(n)$.

Example 4.1. Let $s=2$ and $h=1$. It follows directly from Corollary 4.1 that the exponential generating function of the corresponding Bell numbers is given by

$$
\mathfrak{B e}_{2,1}(x)=e^{1-\sqrt{1-2 x}}
$$

and from Corollary 4.3 that the generalized Bell numbers are given by

$$
\mathfrak{B}_{2,1}(n)=e \sum_{j \geq 0} \frac{(-1)^{n-j} j^{n}}{j !} \prod_{k=0}^{n-1}\left(1-\frac{2 k}{j}\right) .
$$

Example 4.2. Let $s=-1$ and $h=1$. It follows directly from Corollary 4.1 that the exponential generating function of the corresponding Bell numbers is given by

$$
\mathfrak{B}_{-1,1}(x)=e^{x+\frac{1}{2} x^{2}}
$$

and from Corollary 4.2 that the generalized Bell numbers are given by

$$
\mathfrak{B}_{-1,1}(n)=\frac{1}{\sqrt{e}} \sum_{j \geq 0} \frac{(2 j)^{\underline{n}}}{j ! 2^{j}} .
$$

Note that (29) shows that $\mathfrak{B}_{-1,1}(n)$ equals the total number of involutions of $[n]$, upon comparison with Ex. II.13 found on page 122 of [10]. Recall that we derived in Example 3.6 the corresponding generalized Stirling numbers, see (28). Considering the sum over $k$ yields

$$
\sum_{k \geq 0} \mathfrak{S}_{-1 ; 1}(n, k)=\sum_{j \geq 0} \frac{(2 j)^{\underline{n}}}{j ! 2^{j}} \sum_{k \geq j} \frac{1}{2^{k-j}} \frac{(-1)^{k-j}}{(k-j) !}=\sum_{j \geq 0} \frac{(2 j)^{\underline{n}}}{j ! 2^{j}} e^{-\frac{1}{2}}=\mathfrak{B}_{-1,1}(n),
$$

as it should. Let us introduce the Hermite polynomials $H_{n}(z)$ as in [6, Page 50] by their exponential generating function

$$
e^{2 t z-t^{2}}=\sum_{n \geq 0} H_{n}(z) \frac{t^{n}}{n !}
$$

Comparing this with the exponential generating function $\mathfrak{B e}_{-1,1}(x)$ given in (29) shows the very close connection to the Hermite polynomials. Choosing the correspondence $\hat{t}=\frac{i x}{\sqrt{2}}$ and $\hat{z}=\frac{1}{i \sqrt{2}}$, we find

$$
e^{x+\frac{1}{2} x^{2}}=e^{2 \hat{t} \hat{z}-\hat{t}^{2}}=\sum_{n \geq 0} H_{n}(\hat{z}) \frac{\hat{t}^{n}}{n !}=\sum_{n \geq 0} H_{n}\left(\frac{1}{i \sqrt{2}}\right)\left(\frac{i}{\sqrt{2}}\right)^{n} \frac{x^{n}}{n !},
$$


allowing us to conclude that the generalized Bell numbers $\mathfrak{B}_{-1,1}(n)$ are given as special values of Hermite polynomials, i.e.,

$$
\mathfrak{B}_{-1,1}(n)=\left(\frac{i}{\sqrt{2}}\right)^{n} H_{n}\left(\frac{1}{i \sqrt{2}}\right) .
$$

Now we consider the recursion relation for the generalized Bell numbers. Due to the simple explicit expression for the Bell numbers in the case $s=1$, we immediately recognize the recursion relation

$$
\mathfrak{B}_{1 ; h}(n)=(1+(n-1) h) \mathfrak{B}_{1 ; h}(n-1),
$$

the case $h=1$ of which gives $\mathfrak{B}_{1 ; 1}(n)=n !$.

In the case $s=0$, the same procedure as in the conventional case [42, Page 25] works. The exponential generating function is given by $\mathfrak{B e}_{0 ; h}(x)=L_{0 ; h}(x, 1)=e^{\frac{1}{h}\left(e^{h x}-1\right)}$, so that we have

$$
\sum_{n \geq 0} \mathfrak{B}_{0 ; h}(n) \frac{x^{n}}{n !}=e^{\frac{1}{h}\left(e^{h x}-1\right)} .
$$

Taking the logarithm on both sides, differentiating both sides with respect to $x$, multiplying through by $x$ and clearing fractions yields

$$
\sum_{n \geq 1} n \mathfrak{B}_{0 ; h}(n) \frac{x^{n}}{n !}=\left(x e^{h x}\right) \sum_{n \geq 0} \mathfrak{B}_{0 ; h}(n) \frac{x^{n}}{n !},
$$

giving, in analogy to the conventional case, the relation

$$
\mathfrak{B}_{0 ; h}(n)=\sum_{k=0}^{n-1}\left(\begin{array}{c}
n-1 \\
k
\end{array}\right) h^{n-1-k} \mathfrak{B}_{0 ; h}(k) .
$$

Now it remains to consider the case $s \in \mathbb{R} \backslash\{0,1\}$. The exponential generating function of the $\mathfrak{B}_{s ; h}(n)$ is given by $\mathfrak{B e}_{s ; h}(x)=L_{s ; h}(x, 1)$, i.e.,

$$
\sum_{n \geq 0} \mathfrak{B}_{s ; h}(n) \frac{x^{n}}{n !}=e^{\left\{1-(1-h s x)^{\frac{s-1}{s}}\right\} \frac{1}{h(s-1)}} .
$$

Proceeding in the same fashion as in the case $s=0$ above, one obtains

$$
\sum_{n \geq 1} n \mathfrak{B}_{s ; h}(n) \frac{x^{n}}{n !}=\frac{x}{(1-h s x)^{\frac{1}{s}}} \sum_{n \geq 0} \mathfrak{B}_{s ; h}(n) \frac{x^{n}}{n !} .
$$

Using

$$
\frac{x}{(1-h s x)^{\frac{1}{s}}}=x \sum_{m \geq 0}\left(\begin{array}{c}
m+\frac{1}{s}-1 \\
m
\end{array}\right)(h s x)^{m}=\sum_{m \geq 1}\left(\begin{array}{c}
m+\frac{1}{s}-2 \\
m-1
\end{array}\right)(h s)^{m-1} m ! \frac{x^{m}}{m !}
$$


and comparing coefficients for $x^{n}$, we find that

$$
n \mathfrak{B}_{s ; h}(n)=\sum_{k=0}^{n-1}\left(\begin{array}{l}
n \\
k
\end{array}\right) \mathfrak{B}_{s ; h}(k)\left(\begin{array}{c}
n-k+\frac{1}{s}-2 \\
n-k-1
\end{array}\right)(h s)^{n-k-1}(n-k) !
$$

which is equivalent to

$$
\mathfrak{B}_{s ; h}(n)=\sum_{k=0}^{n-1}\left(\begin{array}{c}
n-1 \\
k
\end{array}\right)\left(\begin{array}{c}
n-k+\frac{1}{s}-2 \\
n-k-1
\end{array}\right)(h s)^{n-k-1}(n-k-1) ! \mathfrak{B}_{s ; h}(k) .
$$

Since

$$
(n-k-1) !\left(\begin{array}{c}
n-k+\frac{1}{s}-2 \\
n-k-1
\end{array}\right)=s^{-(n-k-1)} \prod_{j=0}^{n-k-2}(1+j s),
$$

we have finally found the explicit recursion relation

$$
\mathfrak{B}_{s ; h}(n)=\sum_{k=0}^{n-1}\left(\begin{array}{c}
n-1 \\
k
\end{array}\right)\left\{\prod_{j=0}^{n-k-2}(1+j s)\right\} h^{n-1-k} \mathfrak{B}_{s ; h}(k) .
$$

Let us summarize the above observations in the following theorem.

Theorem 4.4. Fix $h \neq 0$. The recursion relation of the generalized Bell numbers is given as follows. If $s \in \mathbb{R} \backslash\{0,1\}$, then

$$
\mathfrak{B}_{s ; h}(n)=\sum_{k=0}^{n-1}\left(\begin{array}{c}
n-1 \\
k
\end{array}\right)\left\{\prod_{j=0}^{n-k-2}(1+j s)\right\} h^{n-1-k} \mathfrak{B}_{s ; h}(k) .
$$

If $s=0$, then

$$
\mathfrak{B}_{0 ; h}(n)=\sum_{k=0}^{n-1}\left(\begin{array}{c}
n-1 \\
k
\end{array}\right) h^{n-1-k} \mathfrak{B}_{0 ; h}(k)
$$

If $s=1$, then

$$
\mathfrak{B}_{1 ; h}(n)=(1+(n-1) h) \mathfrak{B}_{1 ; h}(n-1) .
$$

One can express the recursion relation in a beautiful uniform way.

Proposition 4.5. Fix $h \neq 0$. The recursion relation for the generalized Bell numbers can be written for all $s \in \mathbb{R}$ as

$$
\mathfrak{B}_{s ; h}(n)=\sum_{k=0}^{n-1}\left(\begin{array}{c}
n-1 \\
k
\end{array}\right) \mathfrak{S}_{s ; h}(n-k, 1) \mathfrak{B}_{s ; h}(k) .
$$


Proof. Let us consider first the case $s \neq 0,1$. Recalling the explicit expressions given in Proposition 3.5, we have

$$
\mathfrak{S}_{s ; h}(n-k, 1)=h^{n-k-1} \prod_{j=0}^{n-k-2}(1+j s) .
$$

Inserting this into the recursion relation given in Theorem 4.4 shows the first assertion. If $s=0$, we can use (9) and find $\mathfrak{S}_{0 ; h}(n-k, 1)=h^{n-k-1} S(n-k, 1)=h^{n-k-1}$ so that the asserted recursion above equals the one given in Theorem 4.4. Finally, we consider $s=1$ and observe that the recursion given in Theorem 4.4 can be iterated in the following way:

$$
\begin{aligned}
\mathfrak{B}_{1 ; h}(n) & =\mathfrak{B}_{1 ; h}(n-1)+(n-1) h \mathfrak{B}_{1 ; h}(n-1) \\
& =\mathfrak{B}_{1 ; h}(n-1)+(n-1) h \mathfrak{B}_{1 ; h}(n-2)+(n-1) h(n-2) h \mathfrak{B}_{1 ; h}(n-2) .
\end{aligned}
$$

A small induction shows that this implies $\mathfrak{B}_{1 ; h}(n)=\sum_{l=1}^{n-1} h^{l-1} \frac{(n-1) !}{(n-l) !} \mathfrak{B}_{1 ; h}(n-l)$, or

$$
\mathfrak{B}_{1 ; h}(n)=\sum_{k=1}^{n-1} h^{n-k-1} \frac{(n-1) !}{k !} \mathfrak{B}_{1 ; h}(k) .
$$

Let us now consider the asserted recursion relation (35) for $s=1$. We may use (14) to find $\mathfrak{S}_{1 ; h}(n-k, 1)=(-h)^{n-k-1} s(n-k, 1)=h^{n-k-1}(n-k-1) !$ and, consequently, that

$$
\left(\begin{array}{c}
n-1 \\
k
\end{array}\right) \mathfrak{S}_{1 ; h}(n-k, 1)=\frac{(n-1) !}{k !(n-k-1) !} h^{n-k-1}(n-k-1) !=h^{n-k-1} \frac{(n-1) !}{k !} .
$$

Thus, the asserted recursion relation is equal to the one given in (36), which was shown to be equivalent to the one given in Theorem 4.4.

The recursion relation (35) can be written equivalently in terms of exponential generating functions. Recall that $\mathfrak{S e}_{s ; h}$ is the exponential generating function for the generalized Stirling numbers with $k=1$. Explicit expressions can be found in Proposition 3.6.

Theorem 4.6. Fix $h \neq 0$ and let $s \in \mathbb{R}$. The recursion relation (35) can be written in terms of $\mathfrak{B e}_{s ; h}$ and $\mathfrak{S e}_{s ; h}$ as the differential equation

$$
\mathfrak{B e}_{s ; h}^{\prime}(x)=\mathfrak{S e}_{s ; h}^{\prime}(x) \mathfrak{B} \mathfrak{e}_{s ; h}(x) .
$$

Equivalently, this can be written in integrated form as

$$
\mathfrak{B}_{s ; h}(x)=e^{\mathfrak{S e}_{s ; h}(x)} .
$$

Proof. The proof follows from the recursion relation (35) by multiplying with $\frac{x^{n}}{n !}$, summing over $n$ and manipulating the generating functions. Alternatively, we can check it more directly using expressions already obtained. For example, in the case $s \neq 0,1$, the relation (37) was already derived in (33) due to Proposition 3.6. In the case $s=0$, we 
can write (31) also as $\mathfrak{B e}_{0 ; h}^{\prime}(x)=e^{h x} \mathfrak{B e}_{0 ; h}(x)$ (which is well-known for $h=1$, see [42, Page 45]). Recalling from Proposition 3.6 that $\mathfrak{S e}_{0 ; h}(x):=\frac{1}{h}\left(e^{h x}-1\right)$, we write this as $\mathfrak{B e}_{0 ; h}^{\prime}(x)=\mathfrak{S e}_{0 ; h}^{\prime}(x) \mathfrak{B e}_{0 ; h}(x)$. Finally, in the case $s=1$, the exponential generating function $\mathfrak{B e}_{1 ; h}(x)=\frac{1}{(1-h x)^{1 / h}}$ was determined in Proposition 2.1. According to Proposition 3.6, one has $\mathfrak{S e}_{1 ; h}(x)=\log \left(\frac{1}{(1-h x)^{1 / h}}\right)$, showing directly that $\mathfrak{B e}_{1 ; h}(x)=e^{\mathfrak{S e}_{1 ; h}(x)}($ and, consequently, that $\left.\mathfrak{B e}_{1 ; h}^{\prime}(x)=\mathfrak{S e}_{1 ; h}^{\prime}(x) \mathfrak{B e}_{1 ; h}(x)\right)$.

Note that the case $s=0$ and $h=1$ of (37) yields the classical result

$$
\mathfrak{B e}_{0 ; 1}(x)=e^{e^{x}-1} .
$$

\section{Combinatorial interpretations}

In this section, we provide combinatorial interpretations for the numbers $\mathfrak{S}_{s ; h}(n, k)$. As in the proof of Theorem 3.9 above, it will be more convenient to let $a=h s$ and $b=h-h s$ and then consider the equivalent recurrence

$$
\mathfrak{S}_{a ; b}(n, k)=\mathfrak{S}_{a ; b}(n-1, k-1)+[a(n-1)+b k] \mathfrak{S}_{a ; b}(n-1, k), \quad n \geq k \geq 1,
$$

with $\mathfrak{S}_{a ; b}(0,0)=1$ and $\mathfrak{S}_{a ; b}(n, k)=0$ if $0 \leq n<k$.

When $a=b=1$, we see from (38) that the $\mathfrak{S}_{a ; b}(n, k)$ reduce to the (unsigned) Lah numbers $L(n, k)$ (named for Ivo Lah, see [18] and Example 3.3). It is well known that $L(n, k)=|\mathcal{L}(n, k)|$, where $\mathcal{L}(n, k)$ denotes the set of all distributions of $n$ balls, labeled $1,2, \ldots, n$, among $k$ unlabeled, contents-ordered boxes, with no box left empty. Garsia and Remmel [11] call such distributions Laguerre configurations. See also [33] and [41]. For example, if $n=3$ and $k=2$, then $L(3,2)=6$, the configurations being $\{1,2\},\{3\}$; $\{2,1\},\{3\} ;\{1,3\},\{2\} ;\{3,1\},\{2\} ;\{2,3\},\{1\}$; and $\{3,2\},\{1\}$. The numbers $L(n, k)$ were originally introduced by Lah [18] as the connection constants in the polynomial identities

$$
x(x+1) \cdots(x+n-1)=\sum_{k=0}^{n} L(n, k) x(x-1) \cdots(x-k+1), \quad n \geq 0 .
$$

We observe that the $s=\frac{1}{2}, h=2$ case (equivalently $a=b=1$ ) of the explicit formula in Theorem 3.9 reduces to the well known formula

$$
L(n, k)=\frac{n !}{k !}\left(\begin{array}{c}
n-1 \\
k-1
\end{array}\right), \quad 1 \leq k \leq n,
$$

for the Lah numbers via the binomial identity $\left(\begin{array}{l}n-1 \\ k-1\end{array}\right)=\sum_{j=0}^{k}(-1)^{k-j}\left(\begin{array}{c}k \\ j\end{array}\right)\left(\begin{array}{c}n+j-1 \\ j\end{array}\right)$ (see, e.g., [14]).

Let $L(n)=\sum_{k=0}^{n} L_{n, k}$ and $\mathcal{L}(n)=\cup_{k=0}^{n} \mathcal{L}(n, k)$. Then $L(n)=|\mathcal{L}(n)|$, the cardinality of the set of all distributions of $n$ labeled balls in unlabeled, contents-ordered boxes. The $L(n)$ are analogues of the usual Bell numbers $B(n)$ and are clearly of greater value; see, 
e.g., [27], where they are described as counting sets of lists having size $n$. Letting $s=\frac{1}{2}$, $h=2$ in Corollary 4.2, we obtain a Dobinski formula for $L(n)$, namely

$$
L(n)=\frac{1}{e} \sum_{j \geq 0} \frac{1}{j !}\left\{\prod_{i=0}^{n-1}(j+i)\right\}, \quad n \geq 0,
$$

which does not seem to have been previously noted.

We now proceed in supplying a combinatorial interpretation for the numbers $\mathfrak{S}_{a ; b}(n, k)$ defined by (38) above. To do so, we will now regard $a$ and $b$ as indeterminates and describe statistics on $\mathcal{L}(n, k)$ for which $\mathfrak{S}_{a ; b}(n, k)$ is the joint distribution polynomial.

Definition 5.1. If $\lambda \in \mathcal{L}(n)$ and $i \in[n]$, then we say that $i$ is a record low of $\lambda$ if there are no elements $j<i$ to the left of $i$ within its block in $\lambda$.

For example, if $n=8$ and $\lambda=\{3,1,4\},\{7,5,6,2\},\{8\} \in \mathcal{L}(8)$, then the elements 3 and 1 are record lows in the first block, 7,5 , and 2 are record lows in the second, and 8 is a record low in the third block for a total of six record lows altogether. (For convenience, we will arrange the blocks in ascending order according to the size of the first elements.) Note that the smallest element within a block as well as the left-most one are always record lows.

Definition 5.2. Given $\lambda \in \mathcal{L}(n)$, let rec ${ }^{*}(\lambda)$ denote the total number of record lows of $\lambda$ which are not themselves the smallest member of a block. Let nrec $(\lambda)$ denote the number of elements of $[n]$ which are not record lows of $\lambda$.

For example, if $\lambda$ is as above, then $\operatorname{rec}^{*}(\lambda)=3$ (corresponding to 3,7 , and 5 ) and $\operatorname{nrec}(\lambda)=2$ (corresponding to 4 and 6 ). Given $\lambda \in \mathcal{L}(n)$, let $w(\lambda)=a^{\text {nrec }(\lambda)} b^{r e c}(\lambda)$. If $n \geq k \geq 0$, then define the distribution polynomial $\mathfrak{L}_{a ; b}(n, k)$ by

$$
\mathfrak{L}_{a ; b}(n, k)=\sum_{\lambda \in \mathcal{L}(n, k)} w(\lambda) .
$$

We now give a recurrence for $\mathfrak{L}_{a ; b}(n, k)$. If $\lambda \in \mathcal{L}(n, k)$ and the element $n$ belongs to its own block, then it is counted by neither $r e c^{*}$ nor $n r e c$, and thus the total $w$-weight of all such configurations is $\mathfrak{L}_{a ; b}(n-1, k-1)$. Now suppose that $n$ goes in a block within $\lambda$ with at least one member of $[n-1]$. If $n$ is the left-most member of its block, then it would be a record low that is not the smallest member of its block and hence it would be counted by $\operatorname{rec}^{*}(\lambda)$; thus, the contribution in this case would be $b k \mathfrak{L}_{a ; b}(n-1, k)$. If $n$ is not the left-most member of its block, then it would not be a record low of $\lambda$ and thus it would be counted in $\operatorname{nrec}(\lambda)$, which implies the contribution in this case would be $a(n-1) \mathfrak{L}_{a ; b}(n-1, k)$ since $n$ may directly follow any member of $[n-1]$. Putting together these three cases implies

$$
\mathfrak{L}_{a ; b}(n, k)=\mathfrak{L}_{a ; b}(n-1, k-1)+[a(n-1)+b k] \mathfrak{L}_{a ; b}(n-1, k), \quad n \geq k \geq 1 .
$$

Since $\mathfrak{S}_{a ; b}(n, k)$ clearly satisfies the same boundary conditions as $\mathfrak{L}_{a ; b}(n, k)$, we have shown the following. 
Theorem 5.1. If $n$ and $k$ are non-negative integers, then

$$
\mathfrak{S}_{a ; b}(n, k)=\mathfrak{L}_{a ; b}(n, k) .
$$

Let $\mathfrak{B}_{a ; b}(n)=\sum_{k=0}^{n} \mathfrak{S}_{a ; b}(n, k)$. Using the interpretation above, we may find a combinatorial explanation for the first recurrence in Theorem 4.4, rewritten as

$$
\mathfrak{B}_{a ; b}(n)=\sum_{k=0}^{n-1}\left(\begin{array}{c}
n-1 \\
k
\end{array}\right)\left\{\prod_{j=1}^{n-k-1}(b+j a)\right\} \mathfrak{B}_{a ; b}(k), \quad n \geq 1,
$$

where we have substituted $s=\frac{a}{a+b}$ and $h=a+b$.

Proof. Both sides give the total $w$-weight of all of the members of $\mathcal{L}(n)$, the left-hand side by Theorem 5.1. As for the right-hand side, observe that the $k$-th term of the sum gives the total weight of all of the members of $\mathcal{L}(n)$ in which the cardinality of the block $B$ containing the element $n$ is $n-k$, where $0 \leq k \leq n-1$. To show this, let $B=S \cup\{n\}$, where $|S|=n-1-k$. There are $\left(\begin{array}{c}n-1 \\ n-1-k\end{array}\right)=\left(\begin{array}{c}n-1 \\ k\end{array}\right)$ choices regarding the set $S$ and $L(k)$ ways to arrange the elements of $[n-1]-S$ (which contributes $\mathfrak{B}_{a ; b}(k)$ towards the weight). Finally, the block $B$ contributes $\prod_{j=1}^{n-k-1}(b+j a)\left(^{*}\right)$ for each choice of the set $S$. To see this, suppose the elements of $B$ are $b_{1}<\cdots<b_{n-1-k}<b_{n-k}=n$ and write the element $b_{1}$, noting that it contributes to neither nrec nor ecc $^{*}$ since it is the smallest element of the block. Then write $b_{2}$ either before $b_{1}$ (in which case $b_{2}$ would be a record low) or after $b_{1}$ (in which case it would not). This implies that $b_{2}$ contributes $b+a$ towards the product $(*)$. By the same reasoning, the element $b_{j}, 2 \leq j \leq n-k$, contributes the factor $b+(j-1) a$ towards $(*)$, each one in an independent fashion, which completes the proof.

Using recurrence (38), one can show by induction that the numbers $\mathfrak{S}_{a ; b}(n, k)$ are connection constants in the following polynomial identities, which generalizes (39), but here we will give a combinatorial proof using an interpretation similar to the one given for $\mathfrak{S}_{a ; b}(n, k)$ in Theorem 5.1 above.

Theorem 5.2. The numbers $\mathfrak{S}_{a ; b}(n, k)$ are determined uniquely by the identities

$$
x(x+a) \cdots(x+(n-1) a)=\sum_{k=0}^{n} \mathfrak{S}_{a ; b}(n, k) x(x-b) \cdots(x-(k-1) b), \quad n \geq 0 .
$$

Proof. It suffices to show (43) in the case when $x=\ell b$, where $\ell$ is a positive integer, i.e.,

$$
\ell b(\ell b+a) \cdots(\ell b+(n-1) a)=\sum_{k=0}^{\ell} \mathfrak{S}_{a ; b}(n, k) b^{k} \ell(\ell-1) \cdots(\ell-k+1) .
$$

Given $\ell$ labeled boxes and $n$ labeled balls, let $\mathcal{A}(n, \ell)$ denote the set of distributions of the balls in the boxes, where some of the boxes may be left empty and the balls in each box are ordered. Given $\lambda \in \mathcal{A}(n, \ell)$, let $v(\lambda)=a^{n \operatorname{rec}(\lambda)} b^{\operatorname{rec}(\lambda)}$, where $\operatorname{nrec}(\lambda)$ is defined 
as before and $\operatorname{rec}(\lambda)$ counts all of the record lows of $\lambda$. The product on the left-hand side of (44) then gives the total $v$-weight of all the members of $\mathcal{A}(n, \ell)$, by independently choosing the positions of the $n$ balls in order. To see this, note that ball 1 contributes $b \ell$ since it is automatically a record low in one of the $\ell$ boxes and that ball 2 contributes $b \ell+a$ since it can either follow the first ball in its box (and so contribute $a$ ) or come before it (and thus be a record low in this box) or go in one of the other $\ell-1$ boxes as a record low. Similar considerations show in general that $i$-th ball contributes the factor $b \ell+(i-1) a$ towards the product.

On the other hand, one may form members of $\mathcal{A}(n, \ell)$ by first arranging the balls in unlabeled blocks according to some $\lambda \in \mathcal{L}(n, k)$, where $0 \leq k \leq \ell$, and then placing these blocks in $\ell$ labeled boxes so that no box receives more than one block (which can be done in $\ell(\ell-1) \cdots(\ell-k+1)$ ways). The total weight of all such members of $\mathcal{A}(n, \ell)$ formed is $b^{k} \mathfrak{S}_{a ; b}(n, k) \ell(\ell-1) \cdots(\ell-k+1)$; note that we must multiply by $b^{k}$ to account for the additional record lows which are not included in the $\mathfrak{S}_{a ; b}(n, k)$ factor. Summing over $0 \leq k \leq \ell$ gives the total $v$-weight of $\mathcal{A}(n, \ell)$, which completes the proof.

In [36], it was shown that the Bell numbers satisfy the relation

$$
B(n+m)=\sum_{k=0}^{n} \sum_{j=0}^{m}\left(\begin{array}{l}
n \\
k
\end{array}\right) S(m, j) j^{n-k} B(k), \quad n, m \geq 0,
$$

by an elegant combinatorial argument, which is readily generalized using $w$-weights to yield the following result.

Theorem 5.3. If $n$ and $m$ are non-negative integers, then

$$
\mathfrak{B}_{a ; b}(n+m)=\sum_{k=0}^{n} \sum_{j=0}^{m}\left(\begin{array}{l}
n \\
k
\end{array}\right) \mathfrak{S}_{a ; b}(m, j)\left\{\prod_{i=0}^{n-k-1}(b j+a(i+m))\right\} \mathfrak{B}_{a ; b}(k) .
$$

Proof. Given the set $[n+m]$, one can partition its members into ordered blocks in the following manner. First divide the members of $[m]$ into exactly $j$ subsets in $\mathfrak{S}_{a ; b}(m, j)$ ways. Then choose $k$ members from $[m+1, m+n]=\{m+1, \ldots, m+n\}$ to be partitioned into additional subsets in $\left(\begin{array}{l}n \\ k\end{array}\right) \mathfrak{B}_{a ; b}(k)$ ways. Finally, distribute the remaining $n-k$ members of $[m+1, m+n]$ among the $j$ subsets, which can be done in $\prod_{i=0}^{n-k-1}(b j+a(i+m))$ ways, reasoning as in the prior proofs.

The combinatorial interpretation for $\mathfrak{S}_{a ; b}(n, k)$ given in Theorem 5.1 above readily explains additional relations

Proposition 5.4. If $n$ and $k$ are positive integers, then

$$
\mathfrak{S}_{a ; b}(n, k)=\sum_{j=0}^{k}[a(n-j-1)+b(k-j)] \mathfrak{S}_{a ; b}(n-1-j, k-j) .
$$


Proof. Condition on the largest element, $n-j$, not going in its own block. Note that the elements $1,2, \ldots, n-j-1$ must then occupy $k-j$ blocks, which implies that there are $a(n-j-1)+b(k-j)$ options regarding the placement of $n-j$. Then the elements of $[n-j+1, n]$ must all go in their own singleton blocks.

Proposition 5.5. If $n$ and $k$ are positive integers, then

$$
\mathfrak{S}_{a ; b}(n, k)=\sum_{j=k}^{n}\left\{\prod_{i=j}^{n-1}(a i+b k)\right\} \mathfrak{S}_{a ; b}(j-1, k-1) .
$$

Proof. Assume that the blocks of $\lambda \in \mathcal{L}(n, k)$ are arranged from left to right in ascending order according to the size of the smallest element. Condition on $j$, the smallest element in the right-most block, and note that there are $\prod_{i=j}^{n-1}(a i+b k)$ options regarding placement of the elements of $[j+1, n]$.

As a further application of our combinatorial model, we can find a simple closed form for $\mathfrak{S}_{s ; h}(n, k)$ when $s=-1$ and $h=1$ which was considered above in Example 3.6. Equivalently, we evaluate $\mathfrak{S}_{a ; b}(n, k)$ when $a=-1$ and $b=2$. To do so, we first describe a structure enumerated by $\mathfrak{S}_{a ; b}(n, k)$ in the $a=1, b=2$ case.

Definition 5.3. If $\pi \in \mathcal{L}(n, k)$, then assign each record low of $\pi$ not corresponding to a minimal element in some block one of two colors. No other elements of $\pi$ are colored. If an element $r$ is colored, we will denote it by either $r^{1}$ or $r^{2}$. We will call $\pi$ a colored Laguerre configuration.

Definition 5.4. Let $\mathcal{H}(n, k)$ denote the set of all colored Laguerre configurations of size $n$ having $k$ blocks and $\mathcal{H}(n)=\cup_{k=0}^{n} \mathcal{H}(n, k)$.

For example, if $n=8$ and $k=3$, then $\pi=\left\{2^{1}, 1\right\},\{4,6,8\},\left\{7^{1}, 5^{2}, 3\right\} \in \mathcal{H}(n, k)$. Note that the only members of $\pi$ assigned a superscript are those corresponding to record lows which aren't minimal elements within their respective blocks. From the definitions, we have $|\mathcal{H}(n, k)|=\left.\mathfrak{S}_{a ; b}(n, k)\right|_{a=1, b=2}$.

Define the distribution polynomial $H_{a}(n, k)$ by

$$
H_{a}(n, k)=\sum_{\pi \in \mathcal{H}(n, k)} a^{n r e c(\pi)}
$$

From our combinatorial interpretation of $\mathfrak{S}_{a ; b}(n, k)$, we then have

$$
\mathfrak{S}_{a ; b}(n, k)=H_{-1}(n, k) \quad \text { and } \quad \mathfrak{B}_{a ; b}(n)=\sum_{k=0}^{n} H_{-1}(n, k)
$$

when $a=-1$ and $b=2$.

The following proposition shows that $\mathfrak{S}_{s ; h}(n, k)$ equals the number of involutions of $[n]$ having exactly $k$ cycles when $s=-1$ and $h=1$. 
Proposition 5.6. If $s=-1$ and $h=1$, then

$$
\mathfrak{S}_{-1 ; 1}(n, k)=\left(\begin{array}{c}
n \\
2 k-n
\end{array}\right) \frac{(2 n-2 k) !}{(n-k) ! 2^{n-k}}, \quad 0 \leq k \leq n,
$$

for all $n \geq 0$. Furthermore, $\mathfrak{B}_{-1 ; 1}(n)$ equals the number of involutions of $[n]$.

Proof. From the preceding, we need to show that $H_{-1}(n, k)$ is given by the right side of (48). Denote the set $\mathcal{H}(n, k)$ by $\mathcal{H}$ and let $\mathcal{H}^{+}$and $\mathcal{H}^{-}$denote, respectively, the subsets of $\mathcal{H}$ having even and odd nrec value. To show (48), it suffices to identify a subset $\mathcal{H}^{*} \subseteq \mathcal{H}^{+}$ having cardinality given by the right side, along with an involution of $\mathcal{H}-\mathcal{H}^{*}$ which changes the parity of $n r e c$.

Let $\mathcal{H}^{*}$ consist of all of those members of $\mathcal{H}$ whose blocks are (i) singletons or (ii) doubletons containing elements $a<b$ of the form $\left\{b^{1}, a\right\}$. Note that members of $\mathcal{H}^{*}$ all have zero $n r e c$ value and are synonymous with involutions of $[n]$ having $k$ cycles and thus their number is given by the right side of (48). We now define an involution off of $\mathcal{H}^{*}$ which changes the nrec parity. Let $\pi \in \mathcal{H}-\mathcal{H}^{*}$ and let $\mathcal{B}$ denote the block of $\pi$ containing the smallest element among all the blocks which either have cardinality three or more or are of the form $\left\{b^{2}, a\right\}$ or are of the form $\{a, b\}$, where $a<b$ in the latter two cases. Note that the cases in which $\mathcal{B}$ is of the latter two forms cancel out since the block in the second case has no non-record lows whereas the block in the third has one.

So we may assume $|\mathcal{B}| \geq 3$. Let $a<b<c$ denote the three smallest elements of $\mathcal{B}$. We first exchange the following two cases concerning their relative positions within $\mathcal{B}$ :

$$
c^{2} \cdots b^{1} \cdots a \quad \leftrightarrow \quad b^{1} \cdots a \cdots c
$$

where the other elements of $\mathcal{B}$ are left undisturbed. Note that the ordering of $\mathcal{B}$ on the right has one more non-record low (the $c$ ) than the one of the left, whence the nrec values of the corresponding members of $\mathcal{H}-\mathcal{H}^{*}$ are of opposite parity. Similarly, we exchange the other cases as shown:

$$
c^{1} \cdots b^{1} \cdots a \quad \leftrightarrow \quad b^{1} \cdots c \cdots a
$$

and

$$
b^{2} \cdots a \quad \leftrightarrow \quad a \cdots b
$$

Combining the above mappings yields the desired involution of $\mathcal{H}-\mathcal{H}^{*}$.

Remark 5.7. Equating the expression in (28) with the one given in Proposition 5.6 yields the identity

$$
\sum_{j=\left\lceil\frac{n}{2}\right\rceil}^{k} \frac{(-1)^{k-j}(2 j)^{\underline{n}}}{j !(k-j) !}=\frac{2^{2 k-n} n^{\underline{k}}}{(2 k-n) !}, \quad\left\lceil\frac{n}{2}\right\rceil \leq k \leq n,
$$

for all $n \geq 0$, which we were unable to find in the literature. 
In addition to the above combinatorial interpretation, we can relate the generalized Stirling numbers $\mathfrak{S}_{s ; 1}(n, k)$ for $s \in \mathbb{N}_{0}$ to the $s$-rook numbers introduced by Goldman and Haglund [12]. This connection generalizes the well-known interpretation of the Stirling numbers of second kind to particular rook numbers. In the case $s=0$ - corresponding to the Weyl algebra generated by variables $D, U$ satisfying $D U-U D=1$ - the connection between normal ordering and rook numbers was discussed already by Navon [28]. Varvak pointed out the connection between normal ordering words in variables $D, U$ satisfying $D U-U D=U^{s}$ and $s$-rook numbers in the beautiful paper [40] but, unfortunately, the result given in [40, Theorem 7.1] is not quite correct. Below, we give a corrected version for the particular words $(U D)^{n}$ involving the generalized Stirling numbers $\mathfrak{S}_{s ; 1}(n, k)$, see Proposition 5.9. To begin, we briefly recall that a Ferrers board $B$ is made up of adjacent solid columns of cells with a common lower edge such that the heights (number of cells) $h_{1}, h_{2}, \ldots, h_{n}$ of the columns form a non-decreasing sequence reading from left to right $[12,13,38,40]$. For convenience, we allow columns of height zero. In Figure 1, two examples of Ferrers boards are given with their height vectors $\left(h_{1}, h_{2}, \ldots, h_{n}\right)$.
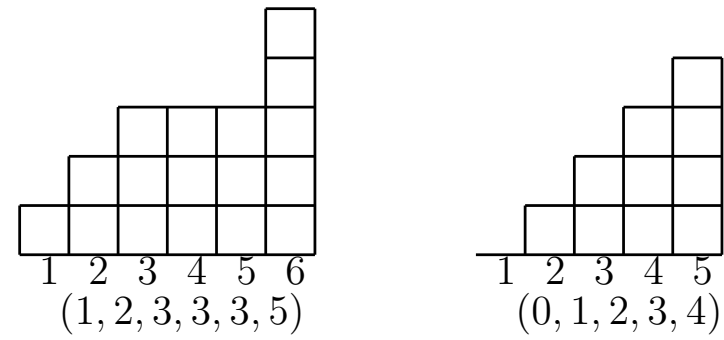

Figure 1: Two examples for Ferrers boards: On the left-hand side, a "typical" board with height vector $(1,2,3,3,3,5)$, and on the right-hand side, the staircase board $J_{5,1}$.

Following [12, 13], Ferrers boards with column heights $(0, m, 2 m, \ldots,(n-1) m)$ are called $m$-jump Ferrers boards and are denoted by $J_{n, m}$. We will use the term staircase board when referring to the particular case $m=1$, i.e., $J_{n, 1}$; see Figure 1 for $J_{5,1}$. If we denote the $k$-th rook number of a Ferrers board $B$ by $r_{k}(B)$, then it is a classical result [13, Page 491] (see also [38, Page 75]) that the Stirling numbers of second kind are rook numbers of the staircase board, i.e.,

$$
S(n, k)=r_{n-k}\left(J_{n, 1}\right) \text {. }
$$

Goldman and Haglund introduced in [12] for any $s \in \mathbb{N}_{0}$ and Ferrers board $B$ so called $s$-rook numbers $r_{k}^{(s)}(B)$ generalizing the conventional case when $s=0$, i.e., $r_{k}^{(0)}(B)=$ $r_{k}(B)$. Let us introduce the notation

$$
x^{(n, m)}:=x(x+m)(x+2 m) \ldots(x+(n-1) m)
$$

for $m>0$ and $x^{(0, m)}=1$. Goldman and Haglund derived as a special case of their 
factorization theorem the following result [12, Example 3] for $m$-jump Ferrers boards

$$
x^{(n, m+s-1)}=\sum_{k=0}^{n} r_{k}^{(s)}\left(J_{n, m}\right) x^{(n-k, s-1)},
$$

which reduces for staircase boards to

$$
x^{(n, s)}=\sum_{k=0}^{n} r_{k}^{(s)}\left(J_{n, 1}\right) x^{(n-k, s-1)} .
$$

Let us turn to the generalized Stirling numbers $\mathfrak{S}_{a: b}(n, k)$ satisfying (43). We restrict to $h=1$ and $s \in \mathbb{N}_{0}$. It follows that $a=s$ and $b=1-s$, so that $\mathfrak{S}_{a ; b}(n, k)$ from (43) with $a=s$ and $b=1-s$ equals $\mathfrak{S}_{s ; 1}(n, k)$ in the notation used in the preceding sections.

Theorem 5.8. Let $h=1$ and $s \in \mathbb{N}_{0}$. The generalized Stirling number $\mathfrak{S}_{s ; 1}(n, k)$ is given as the $(n-k)$-th s-rook number of the staircase board $J_{n, 1}$, i.e.,

$$
\mathfrak{S}_{s ; 1}(n, k)=r_{n-k}^{(s)}\left(J_{n, 1}\right) .
$$

Proof. In the situation considered, equation (43) can be written equivalently as

$$
x^{(n, s)}=\sum_{k=0}^{n} \mathfrak{S}_{s ; 1}(n, k) x^{(k, s-1)},
$$

since $a=s$ and $b=1-s$. Comparing this with (50) shows the assertion.

Let us consider the special case $s=0$ and $h=1$. From Theorem 5.8, we obtain $\mathfrak{S}_{0 ; 1}(n, k)=r_{n-k}^{(0)}\left(J_{n, 1}\right)=r_{n-k}\left(J_{n, 1}\right)$. Combining this with (9), it follows that $S(n, k)=$ $r_{n-k}\left(J_{n, 1}\right)$, as already mentioned in (49). Now let $s=1$ and $h=1$. From Theorem 5.8, we obtain $\mathfrak{S}_{1 ; 1}(n, k)=r_{n-k}^{(1)}\left(J_{n, 1}\right)$. Combining this with (14), it follows that

$$
r_{k}^{(1)}\left(J_{n, 1}\right)=c(n, n-k),
$$

which is exactly ${ }^{1}$ the contents of [12, Theorem 3.1]. In the case $s=2$ and $h=1$, we can use the explicit expression given in [12, Page 520] for $r_{k}^{(2)}\left(J_{n, 1}\right)$ to obtain immediately

$$
\mathfrak{S}_{2 ; 1}(n, k)=\left(\begin{array}{c}
2 n-k-1 \\
2 n-2 k
\end{array}\right) \frac{(2 n-2 k) !}{(n-k) ! 2^{n-k}} .
$$

As a final point, we want to rewrite (7) using Theorem 5.8 and compare it to [40, Theorem 7.1]. For this we first observe that we can write, for $s \in \mathbb{N}_{0}$ and $h=1$,

$$
(V U)^{n}=\sum_{k=0}^{n} r_{n-k}^{(s)}\left(J_{n, 1}\right) V^{s(n-k)+k} U^{k}=\sum_{k=0}^{n} r_{k}^{(s)}\left(J_{n, 1}\right) V^{s k+n-k} U^{n-k} .
$$

Switching to the notation used by Varvak [40], i.e., $(U, V) \rightsquigarrow(D, U)$ (such that $D U-$ $U D=U^{s}$ ), we obtain the following result.

\footnotetext{
${ }^{1}$ Note that there is a typo in the statement of Theorem 3.1 of [12]: It should be $r_{k}^{(1)}\left(J_{n, 1}\right)$ instead of $r_{k}^{(i)}\left(J_{n, 1}\right)$.
} 
Proposition 5.9. Let $w=(U D)^{n}$ be the word of length $2 n$ in the algebra generated by $D, U$ satisfying $D U-U D=U^{s}$ with $s \in \mathbb{N}_{0}$. It is composed of $n D^{\prime} s$ and $n U^{\prime} s$ and one has

$$
(U D)^{n}=\sum_{k=0}^{n} r_{k}^{(s)}\left(J_{n, 1}\right) U^{s k+n-k} D^{n-k},
$$

where $J_{n, 1}$ is the staircase Ferrers board associated to the word $(U D)^{n}$.

Note that this gives a corrected version of [40, Theorem 7.1] for the particular words $w=(U D)^{n}$ (in [40, Theorem 7.1], the summand $s k$ in the exponent is missing).

\section{Conclusion}

In this paper, a thorough discussion of the family of generalized Stirling and Bell numbers introduced recently has been given. For example, the recursion relation of the generalized Stirling numbers and explicit expressions are derived. Furthermore, a combinatorial interpretation is given and it is shown that the generalized Stirling numbers can also be defined as connection coefficients. An alternative interpretation of the generalized Stirling numbers as $s$-rook numbers of staircase Ferrers boards has also been given. The corresponding generalized Bell numbers are defined and many of their properties are derived, for example, the recursion relation, the explicit form of the exponential generating function and a Dobinski-like formula. It is interesting to note that a special case of these considerations yields a Dobinski-like formula for the (unsigned) Lah numbers which seems to be new.

Let us point out some interesting aspects which might warrant further consideration. As a first point, the unimodality of the generalized Stirling numbers should be studied. We conjecture that the sequence $\left\{\mathfrak{S}_{s ; 1}(n, k)\right\}_{k=1, \ldots, n}$ of generalized Stirling numbers is strictly monotone decreasing if $h=1, s \geq 3$ and for $n \geq 3$. A second interesting point would be to obtain a precise asymptotic expression for the generalized Bell numbers. Finally, as a last point we would like to mention that one can define a $q$-deformed version of the generalized Stirling numbers by $(V U)^{n}=\sum_{k>0} \mathfrak{S}_{s ; h}(n, k \mid q) V^{s(n-k)+k} U^{k}$, where the variables $U, V$ satisfy $U V=q V U+h V^{s}$, generalizing the conventional case $s=0$. It is possible that these $q$-deformed generalized Stirling numbers - and the corresponding $q$-deformed generalized Bell numbers - would also possess interesting properties.

\section{References}

[1] H.B. Benaoum, $h$-Analogue of Newton's binomial formula, J. Phys. A: Math. and General 31 (1998) 751-755.

[2] P. Blasiak and P. Flajolet, Combinatorial models of creation-annihilation, arXiv:1010.0354v1 [math.CO].

[3] P. Blasiak, K.A. Penson and A.I. Solomon, The boson normal ordering problem and generalized Bell numbers, Ann. Comb. 7 (2003) 127-139. 
[4] D. Burde, On the matrix equation $X A-A X=X^{p}$, Linear Algebra Appl. 404 (2005) $147-165$.

[5] L. Carlitz, On arrays of numbers, Amer. J. Math. 54 (1932) 739-752.

[6] L. Comtet, Advanced Combinatorics, Reidel, 1974.

[7] G. Dattoli, B. Germano, M.R. Martinelli and P.E. Ricci, Touchard like polynomials and generalized Stirling numbers, arXiv:1010.5934v1 [math.CT].

[8] R. Diaz and E. Pariguan, On the q-meromorphic Weyl algebra, Sao Paulo J. Math. Sci. 3 (2009) 281-296.

[9] B.S. El-Desouky, N.P. Cakić and T. Mansour, Modified approach to generalized Stirling numbers via differential operators, Appl. Math. Lett. 23 (2010) 115-120.

[10] P. Flajolet and R. Sedgewick, Analytic Combinatorics, Cambridge University Press, 2009.

[11] A. Garsia and J. Remmel, A combinatorial interpretation of $q$-derangement and $q$ Laguerre numbers, Europ. J. Combin. 1 (1980) 47-59.

[12] J. Goldman and J. Haglund, Generalized rook polynomials, J. Combin. Theory Ser. A 91 (2000) 509-530.

[13] J.R. Goldman, J.T. Joichi and D.E. White, Rook theory I. Rook equivalence of Ferrers boards, Proc. Amer. Mat. Soc. 52 (1975) 485-492.

[14] R. Graham, D. Knuth and O. Patashnik, Concrete Mathematics: A Foundation for Computer Science, Second Edition, Addison-Wesley, 1989.

[15] A.S. Hegazi and M. Mansour, Some special functions of noncommuting variables, Intern. J. Theor. Phy. 41 (2002) 1815-1825.

[16] F.T. Howard, Degenerate weighted Stirling numbers, Discrete Math. 57 (1985) 45-58.

[17] J. Katriel, Combinatorial aspects of boson algebra, Lett. Nuovo Cimento 10 (1974) $565-567$.

[18] I. Lah, Eine neue Art von Zahlen, ihre Eigenschaften und Anwendung in der Mathematischen Statistik, Mitteilungsbl. Math. Statist. 7 (1955) 203-212.

[19] W. Lang, On generalizations of the Stirling number triangles, J. Integer Seq. 3 (2000) Article 00.2.4.

[20] W. Lang, Combinatorial interpretation of generalized Stirling numbers, J. Integer Seq. 12 (2009) Article 09.3.3.

[21] T. Mansour and M. Schork, The commutation relation $x y=q y x+h f(y)$ and Newton's binomial formula, Ramanujan J., to appear.

[22] M.A. Mendez, P. Blasiak and K.A. Penson, Combinatorial approach to generalized Bell and Stirling numbers and boson normal ordering problem, J. Math. Phys. 46 (2005) Article 083511.

[23] M. Mendez and A. Rodriguez, A combinatorial model for $q$-generalized Stirling and Bell numbers, DMTCS Proc. AJ (2008) 557-570. 
[24] I. Mezö, The r-Bell numbers, J. Integer Seq. 14 (2011) Article 11.1.1.

[25] V.V. Mikhailov, Ordering of some boson operator functions, J. Phys. A: Math. Gen. 16 (1983) 3817-3827.

[26] M. Mohammad-Noori, Some remarks about the derivation operator and generalized Stirling numbers, arXiv:1012.3948v1 [math.CO].

[27] T.S. Motzkin, Sorting numbers for cylinders and other classification numbers, In: Proc. Symp. Pure Math., Vol. 19, American Mathematical Society (1971), 167-176.

[28] A.M. Navon, Combinatorics and fermion algebra, Il Nuovo Cimento 16 (1973) 324330 .

[29] S. Roman, The Umbral Calculus, Dover, 2005.

[30] G.-C. Rota, Finite Operator Calculus, Academic Press, 1975.

[31] H.F. Scherk, De evolvenda functiones (yd.yd.yd...ydX/dx $x^{n}$ ) disquisitiones nonullae analyticae, PhD thesis, Berlin 1823. Publicly available from Göttinger Digitalisierungszentrum (GDZ).

[32] M. Schork, On the combinatorics of normal ordering bosonic operators and deformations of it, J. Phys. A: Math. Gen. 36 (2003) 4651-4665.

[33] M. Shattuck and C. Wagner, Parity theorems for statistics on lattice paths and Laguerre configurations, J. Integer Seq. 8 (2005) Article 05.5.1.

[34] J.-M. Sixdeniers, K.A. Penson and A.I. Solomon, Extended Bell and Stirling numbers from hypergeometric exponentiation, J. Integer Seq. 4 (2001) Article 01.1.4.

[35] N.J.A. Sloane, The On-line Encyclopedia of Integer Sequences, http://oeis.org.

[36] M. Spivey, A generalized recurrence for Bell numbers, J. Integer Seq. 11 (2008) Article 08.2.5.

[37] R.P. Stanley, Differential Posets, J. Amer. Math. Soc. 1 (1988) 919-961.

[38] R.P. Stanley, Enumerative Combinatorics, Volume I, Cambridge University Press, 2002.

[39] R.P. Stanley, Enumerative Combinatorics, Volume II, Cambridge University Press, 2001.

[40] A. Varvak, Rook numbers and the normal ordering problem, J. Combin. Theory Ser. A 112 (2005) 292-307.

[41] C. Wagner, Generalized Stirling and Lah numbers, Discrete Math. 160 (1996) 199218.

[42] H.S. Wilf, generatingfunctionology, A K Peters, 2006. 